TRANSACTIONS OF THE

AMERICAN MATHEMATICAL SOCIETY

Volume 360, Number 10, October 2008, Pages 5265-5289

S 0002-9947(08)04493-0

Article electronically published on April 8, 2008

\title{
TRANSONIC SHOCKS IN 3-D COMPRESSIBLE FLOW PASSING A DUCT WITH A GENERAL SECTION FOR EULER SYSTEMS
}

\author{
SHUXING CHEN
}

\begin{abstract}
This paper is devoted to the study of a transonic shock in threedimensional steady compressible flow passing a duct with a general section. The flow is described by the steady full Euler system, which is purely hyperbolic in the supersonic region and is of elliptic-hyperbolic type in the subsonic region. The upstream flow at the entrance of the duct is a uniform supersonic one adding a three-dimensional perturbation, while the pressure of the downstream flow at the exit of the duct is assigned apart from a constant difference. The problem to determine the transonic shock and the flow behind the shock is reduced to a free boundary value problem of an elliptic-hyperbolic system. The new ingredients of our paper contain the decomposition of the elliptichyperbolic system, the determination of the shock front by a pair of partial differential equations coupled with the three-dimensional Euler system, and the regularity analysis of solutions to the boundary value problems introduced in our discussion.
\end{abstract}

\section{INTRODUCTION}

This paper is devoted to the study of a stationary three-dimensional compressible flow passing a duct with a general section. Assume that the upstream flow at the entrance of the duct is a given supersonic gas flow, which becomes subsonic across a transonic shock front at some place in the duct. Then the whole flow in the duct is expected to be determined. Since in any supersonic flow the upstream part is always not influenced by its downstream part, our problem is to determine the location of the transonic shock and the flow, which is a subsonic one, behind the shock front under some conditions at the lateral wall and the exit of the duct. Such a physical problem often arises in wind tunnel, a nozzle and jet propulsion. Early in 1948 Courant and Friedrichs gave the first systematical analysis on this problem from a mathematical point of view ([10]). Later, T.P.Liu [15, 16], T.P.Liu and H.M.Glaz 11] studied the existence and stability of transonic shock in a quasi one-dimensional case. Recently, G.Q.Chen and M.Feldman [2, 3, Z.Xin and H.Yin [21] also studied the stability of transonic shock by using the model of potential flow equation. In 9] this problem in the model of two-dimensional Euler system is also studied. The new

Received by the editors August 10, 2006.

2000 Mathematics Subject Classification. Primary 35L65; Secondary 35L67.

Key words and phrases. Transonic shock, Euler system, elliptic-hyperbolic system, compressible flow, multidimensional conservation laws.

The paper was partially supported by the National Natural Science Foundation of China 10531020, the National Basic Research Program of China 2006CB805902 and the Doctorial Foundation of National Educational Ministry 20050246001.

(C)2008 American Mathematical Society Reverts to public domain 28 years from publication 
features of the study in this paper are: the flow is governed by a three-dimensional full Euler system, and the duct has a general section, which may not be a rectangle as assumed in 2, 3] and other references. Since the full Euler system is hyperbolic in a supersonic region and is of elliptic-hyperbolic type in a subsonic region, then the related boundary value problem arising in our analysis is more complicated than the problem for potential flow equation in [2, 3, 21]. Meanwhile, because the section of the duct is not a rectangle, we could not make an extension to eliminate the lateral boundary as was done in 2, 9, so that more sophisticated analysis on the regularity and estimates of the solution to related boundary value problems are required. The detailed description of these points will be given later. Besides, due to the arbitrariness of the section of the duct in our study, by using a simple coordinates transformation the result in this paper can also be applied to the case when the lateral boundary of the duct is perturbed.

Physical problems involving transonic shocks often occur in other cases in gas dynamics (e.g. see [4, 6, 8, 10, 20]). Besides, there is also another case for compressible flow passing a duct, where the upstream flow at the entrance is subsonic, while the downstream flow at the exit is supersonic. In this case the flow will be a smooth transonic flow generally, and the equation prescribing the flow becomes a mixed type equation [1, 14, 18, 19.

The flow pattern we are going to study is a perturbation of a background flow, which is a uniform supersonic flow at the entrance that passes across a plane shock through a given point, and then becomes a uniform subsonic flow. The purpose of this paper is to prove the existence and the stability of the perturbed compressible flow including transonic shock under some conditions.

Suppose that the upstream flow is slightly perturbed, and keeping supersonic, then the downstream supersonic flow ahead of any possible shock front is determined. Across a shock, the flow becomes subsonic. Since in a subsonic region any change of flow parameters in the downstream part will influence the whole flow field, then the boundary condition at the exit plays an important role in determining the flow field in the subsonic region, as well as the position of the transonic shock. It seems to be natural to give the pressure at the exit of the duct. However, even in a uniform upstream flow case the pressure at the exit has been determined by the upstream flow. Then in a general perturbed case the pressure of the downstream flow cannot be completely assigned. According to our analysis a reasonable formalation is to assign the pressure of the downstream flow apart from a constant difference. It means that the pressure is given in a space with codimension one. The way to give the boundary condition at the exit is similar to the case for the equi-valued boundary problems of elliptic equations. As a complement of this freedom we let the unknown shock front pass a given point in the duct. Such a restriction is always necessary in dealing with a duct with constant section.

As mentioned in many papers 2, 3, 17, 21 the perturbed supersonic flow ahead of the transonic shock can be easily determined by the data at the entrance. It turns out that we only need to pay attention to determine the flow in the subsonic region, which is located in between the transonic shock front and the exit, and is surrounded by the lateral wall of the duct. To determine the solution of the Euler system in that region, we are led to solve a free boundary value problem of the elliptic-hyperbolic system, where both the flow parameters and the location of the shock front are unknown. The free boundary value problem is solved by using an 
iterative process composed of two steps: solve a fixed boundary value problem in a domain with an approximate shock front as part of its boundary, and update the location of the approximate shock front by using Rankine-Hugoniot conditions. Then the solution can be found by using the fixed point theorem in a suitable Banach space.

To solve the fixed boundary value problem of the Euler system, which is an elliptic-hyperbolic system in the subsonic region, the whole system should be decomposed to a canonical form, in which the elliptic part and the hyperbolic part are separated at the level of the principal part of the system. Due to the complexity of the characteristic varieties for the three-dimensional Euler system we resort to symbolic calculus to complete the decomposition. Moreover, we remark here that in the process of integration of the elliptic part and the hyperbolic part of the system some new difficulties appear and a corresponding technique is required. For instance, to avoid the loss of derivatives in the process of integrating equations we first estimate divergence and rotation of the velocity rather than the velocity itself. Besides, to integrate an elliptic sub-system we have to deal with a boundary value problem of an elliptic equation in a domain with corners, which will generally decrease the regularity of the solution, so that the corresponding estimates are weaker than those in a smooth domain.

Another new ingredient of our analysis is the determination of the function describing the shock front. From two of the Rankine-Hugoniot conditions we obtain derivatives of the function prescribing the shock front with respect to two variables, which leads to an overdetermined system containing two partial differential equations. The two equations defined on the temporarily fixed boundary are coupled with the Euler system defined in the subsonic region. Meanwhile, the condition of solvability of the pair of differential equations leads to a new boundary condition, which will be added to the three remaining Rankine-Hugoniot conditions to determine the solution of the Euler system in the subsonic region. Then the remaining work is to establish all necessary estimates for the corresponding linearized problem and by using iteration to obtain the solution to the original nonlinear problems.

The remaining part of this paper is arranged as follows. In Section 2 we give a mathematical formulation of the physical problem and reduce it to a free boundary problem in the subsonic region with a shock front as its free boundary. In Section 3 we temporarily fix the shock front and then concentrate on solving the fixed boundary value problem. The main work here is to reduce the Euler system to a canonical form, in which the elliptic part and the hyperbolic part are separated at the level of principal part. Accordingly, we obtain corresponding boundary value problems for these two parts, as well as a differential system of first order on shock front. The nonlinear boundary value problem is decomposed in Section 4. Then we derive corresponding linearized sub-problems and establish necessary estimates for the solution to these sub-problems in Section 5. Afterwards, based on these estimates we can solve the nonlinear fixed boundary problem in Section 6. Finally, the nonlinear free boundary problem and the original physical problem are solved in Section 7.

\section{Mathematical Formulation}

Given a duct with constant section $Q=(-1,1) \times \Omega$, where $\Omega$ is a domain in $\mathbb{R}^{2}$ with $C^{2}$ boundary $\partial \Omega$ containing the origin $O$ inside. Consider the motion of 
a compressible flow in the duct $Q$. The motion can be described by the threedmensional Euler system

$$
\begin{aligned}
& \sum_{j=1}^{3} \partial_{x_{j}}\left(\rho u_{j}\right)=0 \\
& \sum_{j=1}^{3} \partial_{x_{j}}\left(\rho u_{k} u_{j}+p \delta_{k j}\right)=0, \quad k=1,2,3, \\
& \sum_{j=1}^{3} \partial_{x_{j}}\left(\rho E u_{j}\right)=0,
\end{aligned}
$$

where $\delta_{k j}=1$ if $k=j$ and $\delta_{k j}=0$ otherwise, $u_{i}$ stands for the velocity component of the flow along the $x_{i}$ axis, and $p, \rho, E$ and $S$ stand for the pressure, density, total energy and entropy respectively. For polytropic gas

$$
\begin{aligned}
p & =(\gamma-1) \exp \left(\frac{S-S_{0}}{c_{\nu}}\right) \rho^{\gamma}, \\
E & =e+\frac{p}{\rho}+\frac{1}{2}|u|^{2}=\frac{\gamma p}{(\gamma-1) \rho}+\frac{1}{2} \rho|u|^{2},
\end{aligned}
$$

where $e=\frac{1}{\gamma-1} \frac{p}{\rho}$ is the internal energy, $|u|^{2}=\sum_{i=1}^{3} u_{i}^{2}$. Obviously, $\rho$ can be written as a function of $p$ and $S$ as $\rho=\rho(p, S)$. For our convenience, we often write $U=\left(u_{1}, u_{2}, u_{3}, p, S\right)^{t}$ to denote the unknown functions in the flow field, and use $a=\left(\frac{\partial p(\rho, S)}{\partial \rho}\right)^{\frac{1}{2}}$ to denote the sound speed.

For $C^{1}$ solutions, the system (2.1)-(2.3) can be written in the following symmetric form:

$$
\sum_{j=1}^{3} A_{j}(U) \partial_{x_{j}} U=0
$$

where

$$
A_{j}(U)=\left(\begin{array}{ccccc}
\rho u_{j} & 0 & 0 & \delta_{j 1} & 0 \\
0 & \rho u_{j} & 0 & \delta_{j 2} & 0 \\
0 & 0 & \rho u_{j} & \delta_{j 3} & 0 \\
\delta_{j 1} & \delta_{j 2} & \delta_{j 3} & \rho^{-1} a^{-2} u_{j} & 0 \\
0 & 0 & 0 & 0 & \rho u_{j}
\end{array}\right), \quad j=1,2,3 .
$$

If $U$ has discontinuity on a surface

$$
\Sigma: \quad x_{1}=f\left(x_{2}, x_{3}\right),
$$

then the Rankine-Hugoniot conditions across $\Sigma$ hold:

$$
\left\{\begin{array}{l}
{\left[\rho u_{1}\right]=\partial_{x_{2}} f\left[\rho u_{2}\right]+\partial_{x_{3}} f\left[\rho u_{3}\right]} \\
{\left[\rho u_{1} u_{2}\right]=\partial_{x_{2}} f\left[\rho u_{2}^{2}+p\right]+\partial_{x_{2}} f\left[\rho u_{2} u_{3}\right],} \\
{\left[\rho u_{1} u_{3}\right]=\partial_{x_{2}} f\left[\rho u_{2} u_{3}\right]+\partial_{x_{3}} f\left[\rho u_{3}^{2}+p\right],} \\
{\left[\rho u_{1}^{2}+p\right]=\partial_{x_{2}} f\left[\rho u_{1} u_{2}\right]+\partial_{x_{3}} f\left[\rho u_{1} u_{3}\right],} \\
{\left[\rho E u_{1}\right]=\partial_{x_{2}} f\left[\rho E u_{2}\right]+\partial_{x_{3}} f\left[\rho E u_{3}\right]}
\end{array}\right.
$$

where $[\cdot]$ denotes the jump of the corresponding quantity across $\Sigma$. 
In this paper we assume that any flow in our study moves basically along the axis of the duct $Q$. The section $x_{1}=-1$ is called the entrance of $Q$, and the section $x_{1}=1$ is called the exit of $Q$. On the lateral wall $(-1,1) \times \partial \Omega$ the flow satisfies the impermeability condition $\vec{u} \cdot \vec{n}=0$. Assume that $U_{b}$ is a gas flow in the duct $Q$, which is a constant supersonic flow $U_{b}^{-}=\left(u_{b 1}^{-}, 0,0, p_{b}^{-}, S_{b}^{-}\right)$at $x_{1}<0$, and becomes a constant subsonic flow $U_{b}^{+}=\left(u_{b 1}^{+}, 0,0, p_{b}^{+}, S_{b}^{+}\right)$across a shock front $S_{0}: x_{1}=0$, where $\left(U_{b}^{-}, U_{b}^{+}\right)$satisfies Rankine-Hugoniot condition (2.9) and the entropy condition $p_{b}^{+}>p_{b}^{-}$. In the whole paper $U_{b}$ is called background flow or background solution. Next we are going to study its three-dimensional perturbation.

Suppose that the supersonic flow $U_{b}^{-}$is perturbed at the entrance of the duct; how does the perturbation influence the flow in the whole duct? It is well known that for the supersonic flow the downstream part can be completely determined by its upstream part. Hence the supersonic flow ahead of the transonic shock can be determined by the data of the upstream flow at the entrance. Assume that the flow also passes a transonic shock through a given point (e.g. the origin) and then becomes a subsonic flow. Then we are required to give a suitable boundary condition at the exit of the duct to determine the subsonic flow in between the transonic flow and the exit.

The main purpose of this paper is to prove the stability of the background solution (or all solutions near to it) under three-dimensional perturbation. It is found that if the pressure of the subsonic flow at the exit is given apart from a constant difference, then in the whole duct a solution of the Euler system with a shock front passing through a fixed point can be determined. Meanwhile, the flow field with transonic shock in the whole duct is slightly perturbed, and their change can be controlled by the perturbation of the data at the entrance. To describe such a conclusion precisely, we formulate the following problem:

$$
(\mathbf{P}): \begin{cases}\text { System (2.6) } & \text { in } Q, \\ \text { Rankine-Hugoniot conditions (2.9) } & \text { on } \Sigma, \\ U=U(-1) & \text { on } x_{1}=-1 \\ p=p_{b}^{+}+g\left(x_{2}, x_{3}\right)+\text { const } & \text { on } x_{1}=1 \\ u_{n}=0 & \text { on }(-1,1) \times \partial \Omega .\end{cases}
$$

Here $u_{n}$ is the normal component of the velocity $u$ on the lateral boundary of the duct, $U(-1)$ is the given data describing a supersonic flow near to $U_{b}^{-}, g\left(x_{2}, x_{3}\right)$ is a given function defined on $\Omega$, and const is an unknown constant, which will be determined with the solution $U$ together.

The main result of this paper is the following theorem.

Theorem 2.1. Assume that $U(-1) \in H^{4}(\Omega)$ satisfies consistence conditions on $\left\{x_{1}=-1,\left(x_{2}, x_{3}\right) \in \partial \Omega\right\}$ and $g\left(x_{2}, x_{3}\right) \in C^{2}(\Omega)$ satisfies $\frac{\partial g}{\partial n}=0$ on $\partial \Omega$. Then the problem $(\mathbf{P})$ has a unique entropy weak solution $\left(U^{-}, U^{+} ; \Sigma\right)$, where $\Sigma$ is a surface defined by the equation $x_{1}=f\left(x_{2}, x_{3}\right)$ with $f(0,0)=0, U^{-}$is defined in $-1<x_{1}<f\left(x_{2}, x_{3}\right),\left(x_{2}, x_{3}\right) \in \Omega$, and $U^{+}$is defined in $f\left(x_{2}, x_{3}\right)<x_{1}<$ $1,\left(x_{2}, x_{3}\right) \in \Omega$. Furthermore, the number const in $(\mathbf{P})$ is also determined, and the 
following estimates hold:

$$
\begin{aligned}
& \left\|U^{-}-U_{b}^{-}\right\|_{C^{1, \alpha}} \leq C_{0} \varepsilon \\
& \left\|U^{+}-U_{b}^{+}\right\|_{C^{1, \alpha}} \leq C_{0} \varepsilon \\
& \|f\|_{C^{2, \alpha}(\Omega)} \leq C_{0} \varepsilon \\
& \mid \text { const } \mid \leq C_{0} \varepsilon
\end{aligned}
$$

where $\alpha$ is a constant determined later, and the constant $C_{0}$ depends only on the background solution and $\alpha$.

The above theorem is also valid in the two-dimensional flow discussed in 9]. In this case the duct becomes a domain in two parallel lines, while the condition at the exit is to determine pressure of the flow up to a constant difference.

As mentioned in Section 1, the solution $U^{-} \in H^{4}$ ahead of the shock front can be easily determined by using the theory of quasilinear hyperbolic system; we only need to concentrate on looking for the solution $U^{+}$in the subsonic region, as well as the location of the shock front $x_{1}=f\left(x_{2}, x_{3}\right)$. Therefore, problem $(\mathbf{P})$ can be reduced to a free boundary value problem as follows:

$$
(\mathbf{F B}): \begin{cases}\text { System (2.6) } & \text { in } Q_{f}: f\left(x_{2}, x_{3}\right)<x_{1}<1, \\ \text { Rankine-Hugoniot conditions (2.9) } & \text { on } \Sigma, \\ p=p_{b}^{+}+g\left(x_{2}, x_{3}\right)+\text { const } & \text { on } x_{1}=1, \\ u_{n}=0 & \text { on }(-1,1) \times \partial \Omega,\end{cases}
$$

where $U^{-}$in (2.9) is given, satisfying $\left\|U^{-}-U_{b}^{-}\right\| \leq \epsilon$. Obviously, once problem (FB) is solved, problem (P) is also solved. Therefore, the proof of Theorem 1 is reduced to

Theorem 2.2. Under the assumption of Theorem 2.1 problem (FB) has a unique solution $U^{+}$defined in $\left.Q_{f}=\left\{x_{2}, x_{3}\right) \in \Omega, f\left(x_{2}, x_{3}\right)<x_{1}<1\right\}$, where

$$
\Sigma: x_{0}=f\left(x_{1}, x_{2}\right), \quad f(0,0)=0
$$

is a part of the boundary of the domain for the unknown functions $U^{+}$. Furthermore, the number const can be determined along with the solution of (FB) and satisfies

$$
\mid \text { const } \mid \leq C_{0} \varepsilon,
$$

and the following estimates of the solution hold:

$$
\begin{aligned}
& \left\|U^{+}-U_{b}^{+}\right\|_{C^{1, \alpha}} \leq C_{0} \varepsilon, \\
& \|f\|_{C^{2, \alpha}} \leq C_{0} \varepsilon
\end{aligned}
$$

with $C_{0}$ depending only on $U_{b}^{ \pm}$and $\alpha$.

The Rankine-Hugoniot conditions (2.9) contain the derivatives of an unknown function $f$. It will be convenient later, if we can give boundary conditions on the shock front without the appearance of the unknown function $f$. Looking at the second and the third equations in (2.9) as a linear algebraic system of $\partial_{x_{2}} f$ and $\partial_{x_{3}} f$, we can solve these partial derivatives as:

$$
\partial_{x_{i}} f=-\Phi_{i} / \Phi_{1}, \quad i=2,3,
$$


where

$$
\begin{aligned}
& \Phi_{1} \triangleq-\left[\rho u_{2}^{2}+p\right]\left[\rho u_{3}^{2}+p\right]+\left[\rho u_{2} u_{3}\right]^{2}, \\
& \Phi_{2} \triangleq\left[\rho u_{1} u_{2}\right]\left[\rho u_{3}^{2}+p\right]-\left[\rho u_{1} u_{3}\right]\left[\rho u_{2} u_{3}\right], \\
& \Phi_{3} \triangleq\left[\rho u_{1} u_{3}\right]\left[\rho u_{2}^{2}+p\right]-\left[\rho u_{1} u_{2}\right]\left[\rho u_{2} u_{3}\right] .
\end{aligned}
$$

Obviously, if $U(-1)$ is near to $U_{b}^{-}$, then the solution $U^{-}$in the supersonic region is also a small perturbation of $U_{b}^{-}$. The fact implies

$$
\Phi_{1} \approx-[p]^{2} \neq 0
$$

so that equation (2.18) makes sense.

Equation (2.18) is employed to modify the approximate shock front. To make the equation solvable the following condition should be satisfied:

$$
\partial_{x_{2}}\left(\frac{\Phi_{3}}{\Phi_{1}}\left(f\left(x_{2}, x_{3}\right), x_{2}, x_{3}\right)\right)=\partial_{x_{3}}\left(\frac{\Phi_{2}}{\Phi_{1}}\left(f\left(x_{2}, x_{3}\right), x_{2}, x_{3}\right)\right) .
$$

Besides, since the velocity on both sides of the shock front is parallel to the boundary, then $f\left(x_{2}, x_{3}\right)$ should satisfy

$$
\frac{\partial f}{\partial n}=0 \quad \text { on } \quad \partial \Omega
$$

which can be verified directly by the Rankine-Hugoniot conditions.

Substituting (2.18) into the other three equations in the Rankine-Hugoniot conditions we obtain

$$
\begin{aligned}
& G_{1}\left(U, U^{-}\right) \triangleq \sum_{j=1}^{3}\left[\rho u_{j}\right] \Phi_{j}=0, \\
& G_{2}\left(U, U^{-}\right) \triangleq \sum_{j=1}^{3}\left[\rho u_{1} u_{j}+p \delta_{j 1}\right] \Phi_{j}=0, \\
& G_{3}\left(U, U^{-}\right) \triangleq \sum_{j=1}^{3}\left[\rho E u_{j}\right] \Phi_{j}=0 .
\end{aligned}
$$

Then the free boundary value problem $(\mathbf{F B})$ can also be written as follows:

$$
\left(\mathbf{F B}_{\mathbf{1}}\right): \begin{cases}\text { System (2.6) } & \text { in } Q_{f}, \\ G_{i}\left(U, U^{-}\right)=0 & \text { on } \Sigma, \quad i=1,2,3, \\ p=p_{b}^{+}+g\left(x_{2}, x_{3}\right)+\text { const } & \text { on } x_{1}=1, \\ u_{n}=0 & \text { on }(-1,1) \times \partial \Omega, \\ f \text { satisfies (2.18) } & , f(0,0)=0 .\end{cases}
$$

We remark here that (2.22) can also be written as

$$
G_{4}\left(U, U^{-}\right) \triangleq-D_{2}\left(\Phi_{3} / \Phi_{1}\right)+D_{3}\left(\Phi_{2} / \Phi_{1}\right)=0,
$$

with

$$
D_{k}:=\partial_{x_{k}}+\left(\partial_{x_{k}} f\right) \partial_{x_{1}}, \quad k=2,3 .
$$

(2.28) (or (2.22) ) is an addtional condition on shock front, and is also the solvability condition on the differential system of first order (2.18). 
Remark 1. For the solution to problem $\left(\mathbf{F B}_{\mathbf{1}}\right)$, the shock front $\Sigma$ is perpendicular to $\partial \Omega$. To prove this fact we denote the normal direction of $\partial \Omega$ by $\vec{n}$, and the two tangential directions of the shock front by $\vec{\tau}_{1}, \vec{\tau}_{2}$. Then denoting the velocity on both sides of $\Sigma$ by $\vec{v}_{ \pm}$, we have

$$
\begin{aligned}
& \vec{v}_{+} \cdot \vec{n}=0=\vec{v}_{-} \cdot \vec{n}, \\
& \vec{v}_{+} \cdot \vec{\tau}_{1}=\vec{v}_{-} \cdot \vec{\tau}_{1}, \\
& \vec{v}_{+} \cdot \vec{\tau}_{2}=\vec{v}_{-} \cdot \vec{\tau}_{2} .
\end{aligned}
$$

Now suppose that $\vec{n}, \vec{\tau}_{1}, \vec{\tau}_{2}$ are linearly independent; then we must have $\vec{v}_{+}=\vec{v}_{-}$, which violates the fact that $\vec{v}$ has discontinuity on the shock front $\Sigma$. Hence, if $\Sigma$ is a real shock front, we must have $\vec{n} \in\left\{\vec{\tau}_{1}, \vec{\tau}_{2}\right\}$. The fact indicates that $\Sigma$ is perpendicular to the boundary of the duct.

\section{Fixed boundary Value problem}

3.1. Reduction to the fixed boundary value problem. To solve the free boundary value problem $\left(\mathbf{F B}_{\mathbf{1}}\right)$ we decompose it to two problems. One is a fixed boundary value problem determining the downstream flow field behind an approximate shock front, the other one is a problem modifying the shape of the shock front. Such an outline has been employed in many papers (e.g. [5, 6, 9]), where the authors also studied free boundary value problems involving shock fronts.

Set

$$
\begin{aligned}
& \Sigma_{\eta}=\left\{f\left(x_{2}, x_{3}\right) \in C^{2, \alpha}(\Omega): f(0,0)=0,\|f\|_{C^{2, \alpha}(\Omega)} \leq \eta\right\}, \\
& O_{\delta}=\left\{U \in C^{1, \alpha}\left(Q_{f}\right):\left\|U-U_{b}^{+}\right\|_{C^{1, \alpha}\left(Q_{f}\right)} \leq \delta\right\}
\end{aligned}
$$

with

$$
\delta<\delta_{0}<1 / 2, \eta<\eta_{0}<1 / 2,
$$

where $\delta_{0}, \eta_{0}$ are small constants depending only on the background solution and will be chosen later. For any $f \in \Sigma_{\eta}$, denote by $S_{f}$ the surface $x_{1}=f\left(x_{2}, x_{3}\right)$. Then we can define a fixed boundary value problem (NL) in $Q_{f}:\left\{f\left(x_{2}, x_{3}\right)<x_{1}<1\right\}$ as follows:

$$
(\mathbf{N L}): \begin{cases}\text { System (2.6) } & \text { in } Q_{f}, \\ G_{i}\left(U, U_{-}\right)=0 & \text { on } \Sigma, \quad i=1,2,3,4, \\ p=p_{b}^{+}+g\left(x_{2}, x_{3}\right)+\text { const } & \text { on } x_{1}=1, \\ u_{n}=0 & \text { on }(-1,1) \times \partial \Omega .\end{cases}
$$

Meanwhile, the problem to update the shock front is

$$
\partial_{x_{i}} f^{\#}=-\Phi_{i} / \Phi_{1}, \quad i=2,3, \quad f^{\#}(0,0)=0 .
$$

(3.5) is a problem for a system of partial differential equations of first order. The equation $G_{4}\left(U, U_{-}\right)=0$ in (3.4) is nothing but the condition of solvability of problem (3.4), so that the problem is solvable.

Later we will show in Section 7 that $f \mapsto f^{\#}$ determines a contract mapping $\mathcal{T}: \Sigma_{\eta} \rightarrow \Sigma_{\eta}$, provided $\varepsilon_{0}$ in Theorem 2.2 is sufficiently small. Then by the fixed point theorem we will obtain a unique fixed point $f$ in $\Sigma_{\eta}$ which is the shock front required in problem $\left(\mathbf{F B}_{\mathbf{1}}\right)$. Correspondingly, the solution $U$ of problem $(\mathbf{N L})$ is exactly the desired subsonic state behind the shock front $S_{f}$. 
3.2. Transformation of the domain. The boundary value problem (NL) is defined in $Q_{f}$, whose left boundary $S_{f}$ is determined by the function $f\left(x_{2}, x_{3}\right)$. To avoid the trouble caused by the change of the location of the boundary $S_{f}$ in the process of seeking the solution to each fixed boundary value problem (NL), we introduce a $C^{2, \alpha}$ homeomorphism to fix the boundary. The homeomorphism is

$$
\Psi_{f}:\left\{\begin{array}{l}
y_{1}=\frac{x_{1}-f\left(x_{2}, x_{3}\right)}{1-f\left(x_{2}, x_{3}\right)} \\
y_{2}=x_{2} \\
y_{3}=x_{3}
\end{array}\right.
$$

which transforms $Q_{f}$ to $Q_{0}=(0,1) \times \Omega$, and transforms the boundary $S_{f}$ and $x_{1}=1$ to $S_{0}: y_{1}=0$ and $S_{1}: y_{1}=1$, respectively.

Obviously,

$$
\left\{\begin{array}{l}
\partial_{x_{1}}=\frac{1}{1-f\left(y_{2}, y_{3}\right)} \partial_{y_{1}} \\
\partial_{x_{i}}=\frac{\left(y_{1}-1\right) \partial_{i} f}{1-f\left(y_{2}, y_{3}\right)} \partial_{y_{1}}+\partial_{y_{i}}, \quad i=2,3 .
\end{array}\right.
$$

Hence the operator $D_{k}$ defined on $y_{1}=0$ by (2.28) is nothing but $\partial_{y_{k}}$.

By using the transformation (3.6), problem (NL) can be written in $(y)$-coordinates. First, system (2.6) is transformed to

$$
\sum_{j=1}^{3} B_{j}(U) \partial_{y_{j}} U=0 \quad \text { in }(0,1) \times \Omega,
$$

where

$$
\left\{\begin{array}{l}
B_{1}(U)=A_{1}(U)+\left(y_{1}-1\right)\left(A_{2}(U) \partial_{2} f+A_{3}(U) \partial_{3} f\right), \\
B_{k}(U)=(1-f) A_{k}(U), \quad k=2,3 .
\end{array}\right.
$$

The boundary conditions on $y_{1}=0, y_{1}=1$ and the lateral wall keep the same form as before. Therefore, we obtain a problem in the $y$-coordinate system, which is still called (NL).

3.3. Decomposition of system. The Euler system (3.8) in the subsonic region is an elliptic-hyperbolic composite system, whose characteristic polynomial has a real eigenvalue with multiplicity 3 and a pair of conjugate complex eigenvalues. To solve the boundary value problem of the composite system (3.8) we will first reduce the system to a canonical form, in which the elliptic part and the hyperbolic part are separated in the principal level.

The symbol of the differential operator in (3.8) is

$$
b(y, \xi)=\sum_{j=1}^{3} B_{j} \xi_{j} .
$$

Here for the notational simplicity we take $\xi_{k}$ as the symbol of $\partial_{k}$ rather than $\frac{1}{\sqrt{-1}} \partial_{k}$. Denote by $\lambda$ the root of $\operatorname{det}\left(\lambda B_{1}-\xi_{2} B_{2}-\xi_{3} B_{3}\right)$, which has one real root $\lambda_{h}$ with multiplicity 3 and two conjugate complex roots

$$
\lambda_{ \pm}=\lambda_{R} \pm \sqrt{-1} \lambda_{I}
$$


Direct calculation shows that

$$
\lambda_{h}(\xi)=\frac{\rho}{\beta}(1-f)\left(\xi_{2} u_{2}+\xi_{3} u_{3}\right),
$$

where

$$
\beta=\rho u_{1}+\left(y_{1}-1\right) \rho\left(u_{2} \partial_{2} f+u_{3} \partial_{3} f\right) .
$$

We notice that $\beta \neq 0$ for small $u_{2}$ and $u_{3}$.

Furthermore,

$$
\lambda_{ \pm}(\xi)=\lambda_{R}(\xi) \pm \sqrt{-1} \lambda_{I}(\xi)
$$

where

$$
\lambda_{R}(\xi)=B / A, \quad \lambda_{I}(\xi)=\sqrt{A C-B^{2}} / A,
$$

with

$$
\begin{aligned}
& A=-1+\beta^{2} /(\rho a)^{2}-\left(y_{1}-1\right)^{2}\left(\left(\partial_{2} f\right)^{2}+\left(\partial_{3} f\right)^{2}\right), \\
& B=B\left(\xi_{2}, \xi_{3}\right)=(1-f)\left(\left(y_{1}-1\right)\left(\partial_{i} f\right) \xi_{i}+\beta u_{i} \xi_{i} /\left(\rho a^{2}\right)\right), \\
& C=C\left(\xi_{2}, \xi_{3}\right)=(1-f)^{2}\left(\left(u_{i} \xi_{i}\right)^{2} / a^{2}-\left(\xi_{2}^{2}+\xi_{3}^{2}\right)\right) .
\end{aligned}
$$

Here and after the same index $i$ in a product means summation for $i=2,3$; for instance, $u_{i} \xi_{i}$ means $u_{2} \xi_{2}+u_{3} \xi_{3}$. Due to $\left(\left(u_{1}\right)_{b}^{+} / a_{b}^{+}\right)^{2}-1<0$ the discriminant $A C-B^{2}$ is positive, so that $\lambda_{I}$ is real-valued provided $\delta_{0}, \eta_{0}$ are small enough.

Corresponding to these eigenvalues the generalized left eigenvectors of (3.10) are

$$
l_{h}^{(i)}(\xi) \quad(i=1,2,3) \text { and } l_{ \pm}(\xi)=l_{R}(\xi) \pm \sqrt{-1} l_{I}(\xi),
$$

where

$$
\begin{aligned}
& l_{h}^{(1)}=\left(\begin{array}{lllll}
-\lambda_{h}(\xi)\left(y_{1}-1\right) \partial_{2} f+(1-f) \xi_{2} & \lambda_{h} & 0 & 0 & 0
\end{array}\right), \\
& l_{h}^{(2)}=\left(\begin{array}{lllll}
-\lambda_{h}(\xi)\left(y_{1}-1\right) \partial_{3} f+(1-f) \xi_{3} & 0 & \lambda_{h} & 0 & 0
\end{array}\right), \\
& l_{h}^{(3)}=\left(\begin{array}{lllll}
0 & 0 & 0 & 0 & 1
\end{array}\right),
\end{aligned}
$$

and

$$
\begin{aligned}
& l_{R}(\xi)=\left((f-1) \rho u_{1}\right)^{-1}\left(\lambda_{R}(\xi)(f-1) \xi_{2}+\left(y_{1}-1\right) \partial_{2} f \lambda_{R}(\xi)\right. \\
& \left.(f-1) \xi_{3}+\left(y_{1}-1\right) \partial_{3} f \lambda_{R}(\xi)(1-f) \rho u_{i} \xi_{i}-\beta \lambda_{R}(\xi) 0\right), \\
& l_{I}(\xi)=\left(\begin{array}{lllll}
\left.(f-1) \rho u_{1}\right)^{-1}\left(\lambda_{I}(\xi)\right. & \left(y_{1}-1\right) \partial_{2} f \lambda_{I}(\xi) & \left(y_{1}-1\right) \partial_{3} f \lambda_{I}(\xi) & -\beta \lambda_{I}(\xi) & 0
\end{array}\right) \text {. }
\end{aligned}
$$

Correspondingly,

$\left(l_{R} B_{1}\right)(\xi)=-\frac{1}{\rho u_{1}}\left(\rho u_{i} \xi_{i} \quad\left(y_{1}-1\right) \partial_{2} f \rho u_{i} \xi_{i}-\beta \xi_{2} \quad\left(y_{1}-1\right) \partial_{3} f \rho u_{i} \xi_{i}-\beta \xi_{3} \quad 0 \quad 0\right)$, so that

$$
\left(l_{R} B_{1}\right)(\partial) U=\partial_{2} u_{2}+\partial_{3} u_{3}-f_{3}(U, f),
$$

where

$f_{3}(U, f)=\sum_{i=2,3} \frac{u_{i}}{u_{1}} \partial_{i} u_{1}+\frac{y_{1}-1}{u_{1}}\left(\partial_{2} f u_{3} \partial_{3} u_{2}-\partial_{3} f u_{3} \partial_{2} u_{2}+\partial_{3} f u_{2} \partial_{2} u_{3}-\partial_{2} f u_{2} \partial_{3} u_{3}\right)$.

Similarly,

$$
\left(l_{I} B_{1}\right)(\xi)=\frac{1}{(1-f) \rho u_{1}}\left(\begin{array}{lllll}
0 & 0 & 0 & A \lambda_{I}(\xi) & 0
\end{array}\right),
$$


so that

$$
\left(l_{I} B_{1}\right)(\partial) U=\frac{A}{(1-f) \rho u_{1}} \lambda_{I}(\partial) p .
$$

By the definition of eigenvectors we have

$$
\left(\begin{array}{c}
l_{R}(\xi) \\
l_{I}(\xi)
\end{array}\right) b(y, \xi)=\left(\begin{array}{cc}
\xi_{1}+\lambda_{R}(\xi) & -\lambda_{I}(\xi) \\
\lambda_{I}(\xi) & \xi_{1}+\lambda_{R}(\xi)
\end{array}\right)\left(\begin{array}{c}
\left(l_{R} B_{1}\right)(\xi) \\
\left(l_{I} B_{1}\right)(\xi)
\end{array}\right) .
$$

Notice that $\ell_{R}(\xi)$ is a polynomial of $\xi$; then the corresponding operator $\ell_{R}(\partial)$ is a differential operator. Meanwhile, all elements of $\ell_{I}(\xi)$ have a common factor $\lambda_{I}(\xi)$, i.e.

$$
\ell_{I}(\xi)=\lambda_{I}(\xi) \hat{\ell}_{I}=\lambda_{I}(\xi)\left((f-1) \rho u_{1}\right)^{-1}\left(1 \quad\left(y_{1}-1\right) \partial_{2} f\left(y_{1}-1\right) \partial_{3} f \quad-\beta \quad 0\right) .
$$

Therefore, we can derive the elliptic part of (3.8) by acting $\ell_{R}(\partial)$ and $\hat{\ell}_{I}$ on (3.8).

Consider the action of $l_{R}(\partial)$ on (3.8). Since both $l_{R}(\partial)$ and $\sum_{j=1}^{3} B_{j} \partial_{j}$ are differential operators, then by using the Leibnitz formula we obtain

$$
\left(\partial_{1}+\lambda_{R}(\partial)\right) v+\frac{1-f}{\rho u_{1}}\left(\partial_{22}+\partial_{33}\right) p+\tilde{f}_{1}(U, f)=0
$$

where

$$
v=\left(l_{R} B_{1}\right)(\partial) U=\partial_{2} u_{2}+\partial_{3} u_{3}-f_{3}(U, f),
$$

with $f_{3}$ being given as (3.15).

The term $\tilde{f}_{1}(U, f)$ in (3.20) is a quadratic form of $u_{2}, u_{3}, \nabla U, f, \nabla f, \nabla^{2} f$ and $\left(U-U_{b}^{+}\right)$, while the degree of $\nabla^{2} f$ is at most 1 . Such terms can be estimated by $C\left(\delta^{2}+\delta \eta\right)$. Moreover, the difference $U^{-}-U_{b}^{-}$is measured by $\epsilon$, whose smallness plays the basic role to ensure the convergence of the iterative process. Later on, all such small terms dominated by $C\left(\delta^{2}+\delta \eta+\epsilon\right)$ will be simply called "good terms". For instance, $\tilde{f}_{1}(U, f)$ in (3.10) and $f_{3}(U, f)$ in (3.15) are both good small terms.

Let $\lambda_{1}>0$ denote the value of $\frac{1-f}{\rho u_{1}}$ at $f=0, U=U_{b}^{+}$. Then (3.20) can be rewritten as

$$
\partial_{1} v+\lambda_{1}\left(\partial_{22}+\partial_{33}\right) p+f_{1}(U, f)=0,
$$

where $f_{1}$ is also a small good term.

Now consider the action of $\hat{l}_{I}$ on (3.8). From (3.18) we have

$$
\hat{\ell} \sum_{j=1}^{3} B_{j} \partial_{j}=\left(l_{R} B_{1}\right)(\partial) U+\hat{\ell} B_{1} \cdot\left(\partial_{1}+\lambda_{R}(\partial)\right) U,
$$

which implies

$$
v+\frac{A}{(1-f) \rho u_{1}}\left(\partial_{1}+\lambda_{R}(\partial)\right) p=0
$$

Let $\lambda_{2}>0$ denote the value of $\frac{-A}{(1-f) \rho u_{1}}$ at $f=0, U=U_{b}^{+}$. Then (3.24) can be written as

$$
v-\lambda_{2} \partial_{1} p=f_{2}(U, f)
$$


where

$$
f_{2}(U, f)=-\frac{A}{(1-f) \rho u_{1}} \lambda_{R}(\partial) p-\left(\lambda_{2}+\frac{A}{(1-f) \rho u_{1}}\right) \partial_{1} p
$$

is a good small term.

Substituting the expression of $v$ into (3.21) and then taking integration on $\Omega$, we have a relation

$$
\begin{aligned}
& \iint_{\Omega}\left(\lambda_{2} \partial_{1} p+f_{2}(U, f)+f_{3}(U, f)\right) d y_{1} d y_{2} \\
& =\iint_{\Omega}\left(\partial_{2} u_{2}+\partial_{3} u_{3}\right) d y_{1} d y_{2}=\iint_{\partial \Omega} \vec{u} \cdot \vec{n} d y_{1} d y_{2}=0 .
\end{aligned}
$$

We now turn to study the hyperbolic part of system (3.8); we regard the equation of conservation laws of momentum and energy in the Euler system as transport equations. Since directly applying these equations to estimate the components of $U$ other than $p$ may cause loss of regularity, then in the process of estimating velocity we first estimate its rotation and divergence. Such a technique will provide the regularity of velocity as good as that for pressure, so that it is helpful in dealing with the compressible flow in a duct with a general constant section.

The velocity vector $\vec{u}$ can be decomposed to a sum of a rotation free part $\overrightarrow{u_{1}}$ and a divergence free part $\overrightarrow{u_{2}}$. In fact, denote by $\Delta_{0}^{-1}$ the solution operator of the homogeneous Dirichlet problem of the Poisson equation on $\Omega$; then $q=\Delta_{0}^{-1} f$ satisfies

$$
\Delta q=f \quad \text { in } \Omega, \quad q=0 \quad \text { on } \partial \Omega .
$$

Then any vector $\vec{u}$ in $\Omega$ can be decomposed as

$$
\vec{u}=\overrightarrow{u_{a}}+\overrightarrow{u_{b}},
$$

where $\vec{u}_{a}=\nabla \Delta_{0}^{-1}(\operatorname{div} \vec{u}), \quad \vec{u}_{b}=\vec{u}-\vec{u}_{a}$. Obviously, div $\vec{u}_{1}=\operatorname{div} \vec{u}$, so that $\vec{u}_{b}$ is divergence free, while $\overrightarrow{u_{a}}$ is rotation free.

Notice that $d=d i v \vec{u}$ can be obtained from the equation of conservation law of mass:

$$
\operatorname{div} \vec{u}=-\frac{1}{\rho} \vec{u} \cdot \nabla \rho
$$

Moreover, denote $\vec{\omega}=$ rot $\vec{u}=\left(\omega_{1}, \omega_{2}, \omega_{3}\right)$. Then direct computation yields

$$
D \vec{\omega}+(\operatorname{div} \vec{u}) \cdot \omega-\nabla \vec{u} \cdot \vec{\omega}+\nabla\left(\frac{1}{\rho}\right) \times \nabla p=0,
$$

where $\nabla \vec{u}$ is the tensor $\nabla \vec{u}=\left(\nabla u_{1}, \nabla u_{2}, \nabla u_{3}\right)$, and $D$ is the transport operator

$$
\left(u_{1}+\left(y_{1}-1\right) u_{i} \partial_{i} f\right) \partial_{1}+(1-f) u_{i} \partial_{i}
$$

obtained by applying (3.7) to the operator $\sum_{i=1}^{3} u_{i} \partial_{x_{i}}$.

The system (3.29) is an ordinary differential system for $\vec{\omega}$ with coefficients depending on the unknown function $U$. Regarding the coefficients as known (as we will do in the process to look for the solution of a linearized problem in the next section), the system can be integrated by using the data on $y_{1}=0$.

Once $d=\operatorname{div} \vec{u}$ and $\omega=\operatorname{rot} \vec{u}$ are obtained, we can regard the equality of the vector field

$$
\Delta \vec{u}=\nabla d-\nabla \times \omega
$$


as an equation for $\vec{u}$ in $Q_{0}$. Finally, the equation

$$
D s=0
$$

is still available, because its integration is simple and will not cause any loss of regularity.

3.4. The boundary conditions. In this subsection we write the boundary conditions on $S_{0}$ in a form with a linear principal part. This is also a preparation of the linearization in Section 5.

Because of $G_{i}\left(U_{b}^{+}, U_{b}^{-}\right)=0$ for $1 \leq i \leq 3$, the condition $G_{i}\left(U, U^{-}\right)=0$ can be written as

$$
\begin{aligned}
& \nabla_{+} G_{i}\left(U_{b}^{+}, U_{b}^{-}\right) \cdot\left(U-U_{b}^{+}\right) \\
= & \nabla_{+} G_{i}\left(U_{b}^{+}, U_{b}^{-}\right) \cdot\left(U-U_{b}^{+}\right)-\left(G_{i}\left(U, U_{b}^{-}\right)-G_{i}\left(U_{b}^{+}, U_{b}^{-}\right)\right) \\
& +\left(G_{i}\left(U, U_{b}^{-}\right)-G_{i}\left(U, U^{-}\right)\right) \\
\triangleq & g_{i}\left(U, U^{-}\right),
\end{aligned}
$$

where $\nabla_{+} G_{i}\left(U, U^{-}\right)$is the gradient of $G_{i}\left(U, U^{-}\right)$with respect to the variable $U$. Direct computation shows that

$$
\begin{aligned}
& \left.\frac{\partial\left(G_{1}, G_{2}, G_{3}\right)\left(U, U^{-}\right)}{\partial U}\right|_{\left(U_{b}^{+}, U_{b}^{-}\right)} \\
& =\left(\begin{array}{ccccc}
-\rho[p]^{2} & 0 & 0 & -u_{1} \frac{[p]^{2}}{a^{2}} & \frac{[p]^{2} p u_{1}}{C_{\nu} a^{2}} \\
-2 \rho u_{1}[p]^{2} & 0 & 0 & -[p]^{2}\left(1+\left(\frac{u_{1}}{a}\right)^{2}\right) & \frac{[p]^{2} p u_{1}^{2}}{C_{\nu} a^{2}} \\
-[p]^{2}\left(\frac{3}{2} \rho u_{1}^{2}+\frac{\gamma p}{\gamma-1}\right) & 0 & 0 & -\frac{\gamma[p]^{2} u_{1}}{\gamma-1}-\frac{[p]^{2} u_{1}^{3}}{2 a^{2}} & \frac{[p]^{2} p u_{1}^{3}}{2 C_{\nu} a^{2}}
\end{array}\right) .
\end{aligned}
$$

The determinant of the sub-matrix composed by the first, fourth and fifth columns is

$$
\frac{p \rho u_{1}[p]^{6}\left(u_{1}^{2}-a^{2}\right)}{(\gamma-1) C_{\nu} a^{2}}
$$

which is not zero for $U^{-}=U_{b}^{-}, U=U_{b}^{+}$. Therefore, for $U \in \mathcal{O}_{\delta}$ the corresponding sub-matrix of (3.32) is also nonsingular. It turns out one can rewrite (3.33) as

$$
u_{i}-\left(u_{i}\right)_{b}^{+}=h_{i}\left(U, U^{-}\right), \quad i=1,4,5,
$$

where $h_{i}$ are linear combinations of $g_{j}(j=1,2,3)$ with constant coefficients depending only on $U_{b}^{ \pm}$. (3.33) can be employed to determine the initial condition of $u_{1}, \rho, S$ for the linearized problem.

In order to solve $u_{2}, u_{3}$ on the boundary $S_{0}$, we use the equation $G_{4}=0$, which can be written as

$$
D_{3} \Phi_{2}-D_{2} \Phi_{3}=\left(\Phi_{2} D_{3} \Phi_{1}-\Phi_{3} D_{2} \Phi_{1}\right) / \Phi_{1} .
$$

Direct calculation yields

$$
\begin{aligned}
D_{3} \Phi_{2}= & {\left[\rho u_{3}^{2}+p\right] \rho u_{1} D_{3} u_{2}+\left[\rho u_{3}^{2}+p\right] D_{3}\left(\rho u_{1}\right) u_{2}-\left[\rho u_{3}^{2}+p\right] \cdot D_{3}\left(\rho^{-} u_{1}^{-} u_{2}^{-}\right) } \\
& +\left[\rho u_{1} u_{2}\right] D_{3}\left[\rho u_{3}^{2}+p\right]-D_{3}\left(\left[\rho u_{1} u_{3}\right]\left[\rho u_{2} u_{3}\right]\right), \\
D_{2} \Phi_{3}= & {\left[\rho u_{2}^{2}+p\right] \rho u_{1} D_{2} u_{3}+\left[\rho u_{2}^{2}+p\right] D_{2}\left(\rho u_{1}\right) u_{3}-\left[\rho u_{2}^{2}+p\right] \cdot D_{2}\left(\rho^{-} u_{1}^{-} u_{3}^{-}\right) } \\
& +\left[\rho u_{1} u_{3}\right] D_{2}\left[\rho u_{2}^{2}+p\right]-D_{2}\left(\left[\rho u_{1} u_{2}\right]\left[\rho u_{2} u_{3}\right]\right) .
\end{aligned}
$$


Then by denoting

$$
\lambda_{4}=\left(p_{b}^{+}-p_{b}^{-}\right) \rho_{b}^{+} u_{1 b}^{+}
$$

the equality (3.36) can be rewritten as

$$
\partial_{2} u_{3}-\partial_{3} u_{2}=f_{4}\left(U, U^{-}\right) \text {on } S_{0},
$$

where $f_{4}\left(U, U^{-}\right)$is a good small term.

Therefore, $u_{2}, u_{3}$ on $S_{0}$ satisfy the problem

$$
\begin{cases}\partial_{2} u_{2}+\partial_{3} u_{3}=f_{2}+f_{3}+\lambda_{2} \partial_{1} p & \text { in } \Omega, \\ \partial_{2} u_{3}-\partial_{3} u_{2}=f_{4} & \text { in } \Omega, \\ n_{2} u_{2}+n_{3} u_{3}=0 & \text { on } \partial \Omega .\end{cases}
$$

In view of the second equation of (3.39), we can define $\Phi$ by

$$
\frac{\partial \Phi}{\partial y_{2}}=u_{2}, \quad \frac{\partial \Phi}{\partial y_{3}}=u_{3}-\int_{0}^{y_{2}} f_{4}\left(\tau, y_{3}\right) d \tau
$$

Then $\Phi$ satisfies

$$
\partial_{2}^{2} \Phi+\partial_{3}^{2} \Phi=\partial_{3} g_{*}+f_{*},
$$

where $f_{*}=f_{2}+f_{3}+\lambda_{2} \partial_{1} p, g_{*}=\int_{0}^{y_{2}} f_{4}\left(\tau, y_{3}\right) d \tau$. The boundary condition in (3.39) is reduced to

$$
\frac{\partial \Phi}{\partial n}=n_{3} g_{*} .
$$

The problems (3.41) and (3.42) are Neumann problems of the Poisson equation. The solvability condition for the problem is

$$
\int_{\partial \Omega} n_{3} g_{*} d \ell=\iint_{\Omega}\left(\partial_{3} g_{*}+f_{*}\right) d y_{2} d y_{3}
$$

i.e. $\iint_{\Omega} f_{*} d y_{2} d y_{3}=0$, which is nothing but the condition (see (3.27) $)$

$$
\iint_{\Omega} \lambda_{2} \partial_{1} p d y_{2} d y_{3}+\iint_{\Omega}\left(f_{2}+f_{3}\right) d y_{2} d y_{3}=0
$$

Therefore, under condition (3.44) $\Phi$ can be determined up to a constant difference, and then $\left(u_{2}, u_{3}\right)=\nabla \Phi$ is uniquely determined.

From the first three equations in (2.6) we have

$$
D u_{j}=-\frac{1}{\rho} \partial_{x_{j}} p, \quad j=1,2,3
$$

then the transversal derivatives of $u_{i}$ on $S_{0}$ can be estimated by $\nabla p$. On the other hand, all tangential derivatives of $u_{i}$ on $S_{0}$ can be derived by solely using the value of $u_{i}$ on $S_{0}$. Therefore, all first order derivatives of $u_{i}$, as well as the vorticity $\vec{\omega}$ on $S_{0}$, can be determined by using the value of $u_{i}, p, \nabla p$ on $S_{0}$. The value of vorticity on $S_{0}$ is then the initial datum on $S_{0}$ for the system (3.29).

Moreover, we have an equation for $\vec{u}$ in the domain $Q_{0}$ as (3.31). Its boundary condition on $S_{0}$ is of Dirichlet type. The condition on the exit $S_{1}$ is of Neumann type (or oblique derivative is given), because of the transport equation (3.45). As for the condition on the lateral boundary we first have

$$
\vec{u} \cdot \vec{n}=0, \text { on }(0,1) \times \partial \Omega .
$$


Denote by $\tau$ the tangential direction of $\partial \Omega$; then $u_{\tau}, u_{1}$ are two tangential components of $\vec{u}$ on $(0,1) \times \partial \Omega$. Due to (3.46) we have

$$
u_{n}=0, \frac{\partial u_{n}}{\partial \tau}=0, \frac{\partial u_{n}}{\partial y_{1}}=0, \quad \text { on }(0,1) \times \partial \Omega .
$$

Therefore, if $\vec{\omega}=\operatorname{rot} \vec{u}$ is known on $(0,1) \times \partial \Omega$, the derivatives $\frac{\partial u_{\tau}}{\partial n}, \frac{\partial u_{1}}{\partial n}$ are also known. Hence the condition on the lateral boundary for (3.31) is

$$
u_{n}=0, \frac{\partial u_{\tau}}{\partial n} \text { and } \frac{\partial u_{1}}{\partial n} \text { are known } \quad \text { on }(0,1) \times \partial \Omega .
$$

\section{DeComposed FORM OF (NL)}

In this section we first decompose problem (NL) into several coupled subproblems.

I. Elliptic problem for $p$.

The pressure $p$ satisfies an elliptic equation (3.20). On the boundary $S_{1}$ it satisfies the condition assigned in (3.4), and on the boundary $S_{0}$ it satisfies (3.35) with $i=4$ and the integral equality (3.27). As for the condition on the lateral boundary for $p$ we use the transport equations

$$
D u_{i}+\frac{1}{\rho} \frac{\partial p}{\partial x_{i}}=0 \quad(i=1,2,3)
$$

Because of $u_{n}=0$ and $n_{1}=0$ on the lateral boundary of the duct, then $n_{2} u_{2}+$ $n_{3} u_{3}=0$. Hence

$$
\begin{aligned}
& \frac{\partial p}{\partial n}=n_{2} \frac{\partial p}{\partial x_{2}}+n_{3} \frac{\partial p}{\partial x_{3}}=-n_{2} D u_{2}-n_{3} D u_{3} \\
& \quad=u_{2} D n_{2}+u_{3} D n_{3}=\sum_{i, j=2,3} u_{i} u_{j} \partial_{i} n_{j} .
\end{aligned}
$$

The right hand side of the above equality is a second order small quantity, which is denoted by $f_{5}(U, f)$, and can be treated as the term $f_{1}(U, f)$. Therefore, the boundary value problem of the elliptic equation in $Q^{+}$for the function $p$ is

$$
\left(\mathbf{P}_{\mathbf{p}}\right): \begin{cases}\lambda_{2} \partial_{11} p+\lambda_{1}\left(\partial_{22}+\partial_{33}\right) p=f_{1}(U, f)-\partial_{1} f_{2}(U, f) & \text { in } Q^{+} \\ p-p_{b}^{+}=h_{4}\left(U, U_{-}\right) & \text {on } S_{0} \\ p-p_{b}^{+}=g+\text { const } & \text { on } S_{1}, \\ \int_{S_{0}} \lambda_{2} \partial_{1} p d y_{2} d y_{3}=-\int_{S_{0}}\left(f_{2}(U, f)+f_{3}(U, f)\right) d y_{2} d y_{3}, & \\ \frac{\partial p}{\partial n}=f_{n}(U, f) . & \end{cases}
$$

We remark here that the compatibility conditions on $\{0\} \times \partial \Omega$ hold because the system and all boundary conditions are nothing but the linear combinations of equalities in the Euler system, the Rankine-Hugoniot conditions on $S_{0}$ and the boundary condition $\vec{n} \cdot \vec{u}=0$ on the lateral boundary, while these conditions are compatible at $\{0\} \times \partial \Omega$. 
II. Determine the velocity on $S_{0}$.

Regard $\overrightarrow{u_{*}}=\left(u_{2}, u_{3}\right)$ as a vector field defined on $S_{0}$. Then (3.39) can be written as

$$
\begin{cases}\operatorname{div} \overrightarrow{u_{*}}=f_{*}(U, f) & \text { in } \Omega, \\ \text { rot } \overrightarrow{u_{*}}=f_{4}(U, f) & \text { in } \Omega, \\ u_{* n}=0 & \text { on } \partial \Omega,\end{cases}
$$

where $f_{*}(U, f)=f_{3}(U, f)+f_{2}(U, f)+\lambda_{2} \partial_{1} p$. Looking at $f_{4}(U, f)$ as a function of $\left(y_{2}, y_{3}\right)$, define $g_{*}=-\int_{0}^{y_{2}} f_{4}\left(\tau, y_{3}\right) d \tau$ as in Section 3. Then we solve a Neumann problem of the Poisson equation

$$
\left(\mathbf{P}_{*}\right):\left\{\begin{array}{l}
\Delta \Phi=\partial_{3} g_{*}+f_{*} \quad \text { in } \Omega, \\
\frac{\partial \Phi}{\partial n}=n_{3} g_{*} \quad \text { on } \partial \Omega, \\
\Phi(0,0)=0 .
\end{array}\right.
$$

Here the solvability of $\left(\mathbf{P}_{*}\right)$ is ensured by (3.44). Once $\Phi$ is obtained, we can define $\left(u_{2}, u_{3}\right)=\nabla \Phi-\left(0, g_{*}\right)$ and obtain the solution of (4.2).

III. Determine the vorticity $\vec{\omega}$ in $\Omega$.

The value of the velocity $\vec{\omega}$ on $S_{0}$ can be determined according to the method described at the end of Section 3. Indeed, $u_{1}=\left(u_{1}\right)_{b}^{+}+h_{1}\left(U, U_{-}\right)$is from (3.33), and $u_{2}$ and $u_{3}$ are given by $\left(\mathbf{P}_{*}\right)$; then $\partial_{y_{2}} \vec{u}, \partial_{y_{3}} \vec{u}$ on $S_{0}$ are determined. Moreover, $\partial_{y_{1}} \vec{u}$ can be determined by (3.45). Therefore, the initial data of $\vec{u}$ on $S_{0}$ is obtained. Then we can solve the solution $\vec{\omega}$ of the problem

$$
\left(\mathbf{P}_{\omega}\right):\left\{\begin{array}{l}
\text { system }\left(\overrightarrow{3.29)} \text { in } Q^{+},\right. \\
\text {initial condition } \vec{\omega}=\operatorname{rot} \vec{u} \text { on } S_{0} .
\end{array}\right.
$$

IV. Determine the velocity of $\vec{u}$ and $S$ in $Q^{+}$.

The value of the entropy $S$ on $S_{0}$ is given by (3.33). Then $S$ in the domain $Q^{+}$ is determined by

$$
\left(\mathbf{P}_{\mathbf{s}}\right):\left\{\begin{array}{l}
D S=0 \text { in } Q^{+} \\
S-\left(S_{b}\right)^{+}=h_{5}\left(U, U_{-}\right) \text {on } S_{0}
\end{array}\right.
$$

The velocity $\vec{u}$ in $Q^{+}$is the solution of the problem

$$
\left(\mathbf{P}_{\mathbf{u}}\right):\left\{\begin{array}{l}
\Delta \vec{u}=\nabla\left(-\frac{1}{\rho} \nabla \rho \cdot \vec{u}\right)-\nabla \times \omega \text { in } Q^{+} \\
\vec{u} \text { is given on } S_{0} \\
D \vec{u}=-\frac{1}{\rho} \nabla p \text { on } S_{1} \\
u_{n}=0, \frac{\partial u_{\tau}}{\partial n} \text { and } \frac{\partial u_{1}}{\partial n} \text { are given } \quad \text { on }(0,1) \times \partial \Omega,
\end{array}\right.
$$

where the equation comes from (3.31), and the boundary condition on the lateral boundary are (3.48). Since $\vec{n}, \vec{\tau}$ in (4.6) stand for the normal direction and the tangential direction on the lateral boundary, then in order to solve problem $\left(\mathbf{P}_{\mathbf{u}}\right)$ we have to use domain decomposition and partition of unity. The details will be given later. 


\section{LineARized FORM OF (NL) AND RELATED ESTIMATES}

The linearized form of (NL) consists of the linearization of $\left(\mathbf{P}_{\mathbf{p}}\right),\left(\mathbf{P}_{*}\right),\left(\mathbf{P}_{\omega}\right),\left(\mathbf{P}_{\mathbf{s}}\right)$ and $\left(\mathbf{P}_{\mathbf{u}}\right)$. Notice that all these nonlinear sub-problems have been written as linearlike forms, i.e. each equation keeps its principal linear part on the left hand side and leaves all nonlinear higher order terms on the right hand side. Then by simply assuming all unknowns $U$ and $f$ on the right hand side be given, we obtain corresponding linearized problems. The corresponding linearized problems are as follows:

$$
\left(\mathbf{P}_{\mathbf{p}}\right)^{\prime}: \begin{cases}\lambda_{2} \partial_{11} p+\lambda_{1}\left(\partial_{22}+\partial_{33}\right) p=\hat{f}_{1} & \text { in } Q^{+}, \\ p-p_{b}^{+}=\hat{h}_{4} & \text { on } S_{0}, \\ p-p_{b}^{+}=g+\text { const } & \text { on } S_{1}, \\ \int_{S_{0}} \lambda_{2} \partial_{1} p d y_{2} d y_{3}=\hat{c}, & \\ \frac{\partial p}{\partial n}=\hat{f}_{n} & \text { on }(0,1) \times \partial \Omega\end{cases}
$$

where $\hat{c}$ is a given constant, while "const" in the third equation is a constant to be determined together with $p$. Then

$$
\left(\mathbf{P}_{*}\right)^{\prime}:\left\{\begin{array}{l}
\Delta \Phi=\partial \hat{g}_{*}+\hat{f}_{*} \quad \text { in } \Omega \\
\frac{\partial \Phi}{\partial n}=n_{3} \hat{g}_{*} \quad \text { on } \partial \Omega \\
\Phi(0,0)=0
\end{array}\right.
$$

and $\left(u_{2}, u_{3}\right)=\nabla \Phi$.

Similarly, we derive the linearization of $\left(\mathbf{P}_{\omega}\right),\left(\mathbf{P}_{\mathbf{s}}\right)$ and $\left(\mathbf{P}_{\mathbf{u}}\right)$ as follows:

$$
\begin{aligned}
& \left(\mathbf{P}_{\omega}\right)^{\prime}:\left\{\begin{array}{l}
\text { system }(\overrightarrow{3.29}) \text { in } Q^{+}, \\
\vec{\omega}=\overrightarrow{\omega_{0}} \text { on } S_{0},
\end{array}\right. \\
& \left(\mathbf{P}_{\mathbf{s}}\right)^{\prime}:\left\{\begin{array}{l}
D S=0 \text { in } Q^{+}, \\
S-\left(S_{b}\right)^{+}=\hat{h}_{5} \text { on } S_{0},
\end{array}\right. \\
& \left(\mathbf{P}_{\mathbf{u}}\right)^{\prime}:\left\{\begin{array}{l}
\Delta \vec{u}=\tilde{f} \text { in } Q^{+}, \\
\vec{u}=\overrightarrow{d_{0}} \text { on } S_{0}, \\
D \vec{u}=\overrightarrow{d_{1}} \text { on } S_{1}, \\
u_{n}=0, \frac{\partial u_{\tau}}{\partial n}=\frac{\partial u_{1}}{\partial n}=0 \text { on }(0,1) \times \partial \Omega .
\end{array}\right.
\end{aligned}
$$

To solve (5.5) we should use the localization method. Let $\left\{\Omega_{\nu}\right\}$ be an open covering of $\bar{\Omega}$ as $\bigcup_{\nu=1}^{\nu_{0}} \Omega_{\nu} \supset \bar{\Omega}$, where each $\Omega_{\nu}$ is an open set with smooth boundary. Let $\left\{\eta_{\nu}\right\}$ be a corresponding partition of unity, i.e. $\eta_{\nu} \in C_{c}^{\infty}\left(\Omega_{\nu}\right)$ for each $\nu$, and $\sum_{\nu=1}^{\nu_{0}} \eta_{\nu} \equiv 1$ on $\bar{\Omega}$. For our convenience we may also let each $\eta_{\nu}$ satisfy $\frac{\partial \eta_{\nu}}{\partial n}=0$. 
Now if $\vec{u}$ is the solution of (5.5), then $\vec{u}_{\nu}=\eta_{\nu} \vec{u}$ satisfies

$$
\left(\mathbf{P}_{\mathbf{u}}\right)_{\nu}^{\prime}:\left\{\begin{array}{l}
\Delta \overrightarrow{u_{\nu}}=\eta_{\nu} \tilde{f}-\left(\Delta \eta_{\nu}\right) \vec{u}-2 \sum_{j=1}^{3}\left(\eta_{\nu}\right)_{y_{j}}(\vec{u})_{y_{j}} \text { in } Q^{+}, \\
\overrightarrow{u_{\nu}}=\eta_{\nu} \overrightarrow{d_{0}} \text { on } S_{0}, \\
D \overrightarrow{u_{\nu}}=\eta_{\nu} \overrightarrow{d_{1}} \text { on } S_{1}, \\
\left(u_{\nu}\right)_{n}=0, \frac{\partial\left(u_{\nu}\right)_{\tau}}{\partial n}=\frac{\partial\left(u_{\nu}\right)_{1}}{\partial n}=0 \text { on }(0,1) \times \partial \Omega .
\end{array}\right.
$$

Furthermore, by introducing the coordinate transformation $\tau_{\nu}$, problem $\left(\mathbf{P}_{\mathbf{u}}\right)_{\nu}^{\prime}$ can be reduced to a problem for $\vec{v}_{\nu}=\vec{u}_{\nu} \circ \tau_{\nu}^{-1}$ in $\tau_{\nu}\left(\Omega_{\nu} \cap \tilde{\Omega}\right)$. This is a standard boundary value problem of the elliptic equation for the component of $\vec{v}_{\nu}$. Finally, when we solve $\vec{v}_{\nu}$, then $\vec{u}_{\nu}=\vec{v}_{\nu} \circ \tau_{\nu}$ is also obtained.

In order to solve (NL) via its linearization, we also have to derive a series of estimates for the solution to the linearized problem (5.1)-(5.6). The basic estimates are given in a $C^{1, \alpha}$ Hölder space, though the regularity estimates can be improved in some cases. In the sequel the notation $f \in C^{-1, \alpha}$ with $\alpha>0$ means $f=\sum_{i} \partial_{i} f_{i}$ with all $f_{i} \in C^{0, \alpha}$. Meanwhile, $\|f\|_{C^{-1, \alpha}}$ means the sum $\sum_{i}\left\|f_{i}\right\|_{C^{0, \alpha}}$.

For problem $\left(\mathbf{P}_{\mathbf{p}}\right)^{\prime}$, we define $p_{g}$ and $p_{c}$ as the solutions to

$$
\begin{cases}\lambda_{2} \partial_{11} p_{g}+\lambda_{1}\left(\partial_{22}+\partial_{33}\right) p_{g}=\hat{f}_{1} & \text { in } Q^{+}, \\ p_{g}-p_{b}^{+}=h_{4} & \text { on } S_{0}, \\ p_{g}-p_{b}^{+}=g & \text { on } S_{1}, \\ \frac{\partial p_{g}}{\partial n}=0 & \text { on }(0,1) \times \partial \Omega\end{cases}
$$

and

$$
\begin{cases}\lambda_{2} \partial_{11} p_{c}+\lambda_{1}\left(\partial_{22}+\partial_{33}\right) p_{c}=0 & \text { in } Q^{+}, \\ p_{c}=0 & \text { on } S_{0}, \\ p_{c}=1 & \text { on } S_{1}, \\ \frac{\partial p_{c}}{\partial n}=0 & \text { on }(0,1) \times \partial \Omega .\end{cases}
$$

Because $p_{c}$ is not a trivial solution, then $\frac{\partial p_{c}}{\partial n}>0$ on $S_{1}$ according to the Hopf maximum principle. Therefore, we can take $p=p_{g}+c_{0} p_{c}$, where

$$
c_{0}=\frac{\frac{1}{\lambda_{2}} \hat{c}-\int_{S_{0}} \partial_{1} p_{g} d y_{2} d y_{3}}{\int_{S_{0}} \partial_{1} p_{c} d y_{2} d y_{3}} .
$$

Obviously, $p=p_{g}+c_{0} p_{c}$ satisfies $\left(\mathbf{P}_{\mathbf{p}}\right)^{\prime}$, and the constant const in (5.1) takes the value $c_{0}$. By using the theory of elliptic equations in a domain with piecewise smooth boundary (see [12, 13]) we have

$\|p\|_{C^{1, \alpha}\left(Q^{+}\right)} \leq C\left(\left\|\hat{f}_{1}\right\|_{C^{-1, \alpha}\left(Q^{+}\right)}+\left\|h_{4}\right\|_{C^{1, \alpha}\left(S_{0}\right)}+\|g\|_{C^{1, \alpha}\left(S_{1}\right)}+\left\|\hat{f}_{n}\right\|_{C^{0, \alpha}((0,1) \times \partial \Omega)}+|\hat{c}|\right)$, while the constant $c_{0}$ can also be controlled by the right hand side of (5.10).

For problem $\left(\mathbf{P}_{*}\right)^{\prime}$ we first have estimate (see [12])

$$
\|\Phi\|_{C^{1, \alpha}(\Omega)} \leq C\left(\left\|\hat{g}_{*}\right\|_{C^{\alpha}(\Omega)}+\left\|\hat{f}_{*}\right\|_{C^{\alpha}(\Omega)}\right)
$$


then

$$
\left\|\overrightarrow{u_{*}}\right\|_{C^{\alpha}(\Omega)} \leq C\left(\left\|\hat{g}_{*}\right\|_{C^{\alpha}(\Omega)}+\left\|\hat{f}_{*}\right\|_{C^{\alpha}(\Omega)}\right) .
$$

Since the function $\hat{g}_{*}$ depends on the first derivatives of $U, f$, then applying the estimate (5.11) to (4.2), one will meet the difficulty of loss of derivatives. To get rid of it we have to derive an estimate of $\left\|\overrightarrow{u_{*}}\right\|_{C^{1, \alpha}(\Omega)}$ by using the right hand side of (5.11). To this end we directly use (4.2) rather than (4.3)

Let $1 \equiv \Sigma \eta_{\nu}$ be a partition of unity, $\vec{u}=\Sigma \eta_{\nu} \overrightarrow{u_{*}}=\Sigma \vec{u}_{* \nu}$. Then $\vec{u}_{* \nu}$ satisfies

$$
\begin{cases}\operatorname{div} \vec{u}_{* \nu}=\eta_{\nu} \hat{f}_{*}-\nabla \eta_{\nu} \overrightarrow{u_{*}} & \text { in } \Omega, \\ \operatorname{rot} \overrightarrow{u_{* \nu}}=\eta_{\nu} \hat{f}_{4}-\nabla \eta_{\nu} \overrightarrow{u_{*}} & \text { in } \Omega, \\ \vec{n} \cdot \vec{u}_{* \nu}=0 & \text { on } \partial \Omega .\end{cases}
$$

Fix $\nu$ and simply denote $\vec{u}_{* \nu}$ by $\left(v_{2}, v_{3}\right)$ in a given coordinate system $O z_{2} z_{3}$, which can be a rotation of $\mathrm{Oy}_{2} y_{3}$; then the equations in (5.12) are

$$
\left\{\begin{array}{l}
\frac{\partial v_{2}}{\partial z_{2}}+\frac{\partial v_{3}}{\partial z_{3}}=g_{\nu 1} \\
\frac{\partial v_{2}}{\partial z_{3}}-\frac{\partial v_{3}}{\partial z_{2}}=g_{\nu 2} .
\end{array}\right.
$$

The boundary condition still takes the form

$$
v_{2} n_{2}+v_{3} n_{3}=0 .
$$

It is easy to derive from (5.13)

$$
\begin{aligned}
& \Delta v_{2}=\partial_{z_{2}} g_{\nu 1}+\partial_{z_{3}} g_{\nu 2}, \\
& \Delta v_{3}=\partial_{z_{3}} g_{\nu 1}-\partial_{z_{2}} g_{\nu 2} .
\end{aligned}
$$

Besides, by differentiating the condition $n_{2} v_{2}+n_{3} v_{3}=0$ along $\partial \Omega$ we obtain

$$
-\partial_{z_{2}}\left(n_{2} v_{2}+n_{3} v_{3}\right) n_{3}+\partial_{z_{3}}\left(n_{2} v_{2}+n_{3} v_{3}\right) n_{2}=0 .
$$

By using (5.13) and (5.14) we obtain

$$
\begin{gathered}
-2 n_{2} n_{3} \frac{\partial v_{2}}{\partial z_{2}}+\left(n_{2}^{2}-n_{3}^{2}\right) \frac{\partial v_{2}}{\partial z_{3}}-\frac{\partial n_{2}}{\partial z_{2}} n_{3} v_{2}+\frac{\partial n_{3}}{\partial z_{2}} v_{2} n_{2} \\
+\frac{\partial n_{2}}{\partial z_{3}} n_{2} v_{2}+\frac{\partial n_{3}}{\partial z_{3}} \frac{n_{2}^{2}}{n_{3}} v_{2}+n_{3}^{2} g_{\nu 2}+n_{2} n_{3} g_{\nu 1}=0 .
\end{gathered}
$$

When $n_{3} \neq 0$, the problem (5.15), (5.17) is a regular oblique derivative problem of the Poisson equation; hence the solution $v_{2}$ satisfies the estimate

$$
\left\|v_{2}\right\|_{C^{1, \alpha}(\Omega)} \leq C\left(\left\|g_{\nu 1}\right\|_{C^{\alpha}(\Omega)}+\left\|g_{\nu 2}\right\|_{C^{\alpha}(\Omega)}\right) .
$$

By using the system (5.13) once more we have the estimate for $v_{3}$ :

$$
\left\|v_{3}\right\|_{C^{1, \alpha}(\Omega)} \leq C\left(\left\|g_{\nu 1}\right\|_{C^{\alpha}(\Omega)}+\left\|g_{\nu 2}\right\|_{C^{\alpha}(\Omega)}\right) .
$$

Now if $n_{3}=0$ at some point in the support of $v_{2}$, then by taking each $\Omega_{\nu}$ sufficiently small we have $n_{2} \neq 0$ in this support. Hence we can derive an oblique derivative condition for $v_{3}$ similar to (5.17). Combining it with equation (5.16) we can derive the estimate (5.19) for $\left\|v_{3}\right\|_{C^{1, \alpha}(\Omega)}$ by similarly using the theory of elliptic equations and then by using the system (5.13) to obtain the estimate for $\left\|v_{2}\right\|_{C^{1, \alpha}(\Omega)}$. 
Notice that $\left(v_{2}, v_{3}\right)$ are the components of $\vec{u}_{* \nu}$ in fact. When we establish the estimate $\left\|\vec{u}_{* \nu}\right\|_{C^{1, \alpha}(\Omega)}$ for each $\nu$, then by making summation with respect to $\nu$ and by using the estimate (5.11) we have

$$
\begin{aligned}
& \left\|\overrightarrow{u_{*}}\right\|_{C^{1, \alpha}(\Omega)} \leq C\left(\left\|\hat{f}_{4}\right\|_{C^{\alpha}(\Omega)}+\left\|\hat{f}_{*}\right\|_{C^{\alpha}(\Omega)}+\left\|\overrightarrow{u_{*}}\right\|_{C^{\alpha}(\Omega)}\right) \\
& \quad \leq C\left(\left\|\hat{f}_{4}\right\|_{C^{\alpha}(\Omega)}+\left\|\hat{f}_{*}\right\|_{C^{\alpha}(\Omega)}\right) .
\end{aligned}
$$

For problem $\left(\mathbf{P}_{\mathbf{s}}\right)^{\prime}$ a simple integration gives

$$
\left\|S-S_{b}^{+}\right\|_{C^{1, \alpha}\left(Q^{+}\right)} \leq C\left\|\hat{h}_{5}\right\|_{C^{1, \alpha}\left(Q^{+}\right)} .
$$

For problem $\left(\mathbf{P}_{\omega}\right)^{\prime}$, since the system $(3.29)$ is a homogeneous system, and the coefficients of the derivatives of $\vec{\omega}$ are $C^{1, \alpha}$ smooth, then we can easily obtain $C^{\alpha}$ estimates of $\vec{\omega}$ via the theory of ordinary differential systems. Indeed, the characteristics of the operator $D$ are

$$
y_{2}=\eta_{2}\left(y_{20}, y_{30}, y_{1}\right), \quad y_{3}=\eta_{3}\left(y_{20}, y_{30}, y_{1}\right)
$$

where $y_{20}, y_{30}$ are the values of $y_{2}, y_{3}$ on $S_{0}$, and $\frac{\partial\left(y_{2}, y_{3}\right)}{\partial\left(y_{20}, y_{30}\right)} \neq 0$ in the whole $Q^{+}$. Then by integrating the system of (3.29) along characteristics we obtain

$$
\begin{aligned}
\omega_{k} & =\sum_{j} F_{k j}\left(y_{1}, y_{20}, y_{30}\right) \omega_{j 0} \\
& =\sum_{j} F_{k j}\left(y_{1}, \zeta_{2}\left(y_{1}, y_{2}, y_{3}\right), \zeta_{3}\left(y_{1}, y_{2}, y_{3}\right)\right) \omega_{j 0}\left(y_{2}, y_{3}\right),
\end{aligned}
$$

where $\zeta_{2}, \zeta_{3}$ are the inverse of (5.22), and $F_{k j}(1 \leq k, j \leq 3)$ depend on $U, f$, and are $C^{0, \alpha}$ smooth at least. Furthermore, the estimate

$$
\|\vec{\omega}\|_{C^{0, \alpha}\left(Q^{+}\right)} \leq C\left(\|\vec{\omega}\|_{C^{0, \alpha}\left(S_{0}\right)}+\left\|\zeta_{j}\right\|_{C^{0, \alpha}\left(Q^{+}\right)}\right) \leq\|\vec{u}\|_{C^{1, \alpha}\left(Q^{+}\right)}
$$

holds.

Finally, to obtain the estimate of $\vec{u}$ we can first estimate $\vec{u}_{\nu}$ via problem $\left(\tilde{\mathbf{P}}_{\mathbf{u}}\right)_{\nu}^{\prime}$, and then via transformation and summation establish

$$
\|\vec{u}\|_{C^{1, \alpha}\left(Q^{+}\right)} \leq C\left(\|\tilde{f}\|_{C^{-1, \alpha}\left(Q^{+}\right)}+\left\|\overrightarrow{d_{0}}\right\|_{C^{1, \alpha}\left(S_{0}\right)}+\left\|\overrightarrow{d_{1}}\right\|_{C^{0, \alpha}\left(S_{0}\right)}\right) .
$$

\section{ExistenCE of PROBlem (NL)}

In this section we will establish the existence of the nonlinear fixed boundary problem (NL) for any $f \in \Sigma_{\eta}$. Regarding the decoupled form of (NL) derived in Section 5 as a mapping defined on $C^{1, \alpha}\left(Q^{+}\right)$, we are going to prove that $T$ has a fixed point in $O_{\delta}$, where $\delta \leq \delta_{0}$ is sufficiently small.

For any $f \in \Sigma_{\eta}, U \in O_{\delta}$, the expression of $f_{k}(U, f)$ with $1 \leq k \leq 4$ in (3.22), (3.25), (3.14), (3.38) satisfy

$$
\left\|f_{1}(U, f)\right\|_{C^{-1, \alpha}\left(Q^{+}\right)}+\sum_{k=2}^{4}\left\|f_{k}(U, f)\right\|_{C^{0, \alpha}\left(Q^{+}\right)} \leq C\left(\delta^{2}+\delta \eta+\epsilon\right)=C e,
$$

where $e=\delta^{2}+\eta \delta+\epsilon$, and $C$ is a constant independent of $\delta, \eta, \epsilon$. Meanwhile, $h_{i}\left(U, U^{-}\right)$with $i=1,4,5$ in (3.33) satisfy

$$
\left\|h_{i}\left(U, U^{-}\right)\right\| \leq C\left(\epsilon+\delta^{2}\right) \leq C e .
$$


Then the right hand side of $\left(\mathbf{P}_{\mathbf{p}}\right)$ satisfies

$$
\begin{gathered}
\left\|f_{1}(U, f)-\partial_{1} f_{2}(U, f)\right\|_{C^{-1, \alpha}\left(Q^{+}\right)} \leq C e, \\
\left|\int_{S_{0}}\left(f_{2}(U, f)+f_{3}(U, f)\right) d y_{2} d y_{3}\right| \leq C e .
\end{gathered}
$$

Hence (5.10) yields

$$
\|p\|_{C^{1, \alpha}\left(\Omega_{+}\right)} \leq C e .
$$

Furthermore, the estimate (5.20) implies

$$
\left\|\left(u_{2}, u_{3}\right)\right\|_{C^{1, \alpha}\left(S_{0}\right)} \leq C e,
$$

(5.21) implies

$$
\left\|S-S_{b}^{+}\right\|_{C^{1, \alpha}\left(Q^{+}\right)} \leq C e
$$

and (5.24) implies

$$
\|\vec{\omega}\|_{C^{0, \alpha}\left(Q^{+}\right)} \leq C e .
$$

Here we have employed (6.4) and (3.45).

Finally, we give the estimates on the right hand side of the equation in problem $\left(\mathbf{P}_{\mathbf{u}}\right)$. That is,

$$
\left\{\begin{array}{l}
\left\|\nabla\left(-\frac{1}{\rho} \nabla \rho \cdot \vec{u}\right)\right\|_{C^{-1, \alpha}\left(Q^{+}\right)} \leq C e, \\
\|\nabla \times \vec{\omega}\|_{C^{-1, \alpha}\left(Q^{+}\right)} \leq C e \\
\|u\|_{C^{1, \alpha}\left(S_{0}\right)} \leq C e \\
\left\|-\frac{1}{\rho} \nabla p\right\|_{C^{0, \alpha}\left(S_{1}\right)} \leq C e
\end{array}\right.
$$

Then the inequality (5.25) gives the estimate of $\vec{u}$ :

$$
\|\vec{u}\|_{C^{1, \alpha}\left(Q^{+}\right)} \leq C e .
$$

Let $\delta_{0}, \eta_{0}, \epsilon$ be sufficiently small. We can confirm $T U \in O_{\delta}$ according to above argument, so that $T$ is an inner mapping from $O_{\delta}$ to $O_{\delta}$.

Next we prove that $T$ is also a contractive mapping. Indeed, assume that $U^{(1)}, U^{(2)}$ are two elements in $O_{\delta}$. Letting

$$
\tilde{U} \equiv U^{(1)}-U^{(2)}=\left(u_{1}^{(1)}-u_{1}^{(2)}, u_{2}^{(1)}-u_{2}^{(2)}, u_{3}^{(1)}-u_{3}^{(2)}, p^{(1)}-p^{(2)}, S^{(1)}-S^{(2)}\right),
$$

we derive the equation for $\tilde{U}$ and establish its estimates. For our later convenience we replace the function $f \in \Sigma_{\eta}$ on the right hand side of all inequalities by $f^{(1)}$ or $f^{(2)}\left(f^{(1)}=f^{(2)}=f\right.$ in this section $)$. First $\tilde{p}$ satisfies

$$
\left(\tilde{\mathbf{P}}_{\mathbf{p}}\right): \begin{cases}\lambda_{2} \partial_{11} \tilde{p}+\lambda_{1}\left(\partial_{22}+\partial_{33}\right) \tilde{p}=f_{1}\left(U^{(1)}, f^{(1)}\right)-f_{1}\left(U^{(2)}, f^{(2)}\right) & \\ -\partial_{1} f_{2}\left(U^{(1)}, f^{(1)}\right)+\partial_{1} f_{2}\left(U^{(2)}, f^{(2)}\right) & \text { in } \Omega, \\ \tilde{p}=h_{4}\left(U^{(1)}, U^{-}\right)-h_{4}\left(U^{(2)}, U^{-}\right) & \text {on } S_{0}, \\ \tilde{p}=\text { const } & \text { on } S_{1}, \\ \int_{S_{0}} \lambda_{2} \partial_{1} \partial p d y_{2} d y_{3}=-\int_{S_{0}}\left(f_{2}\left(U^{(1)}, f^{(1)}\right)-f_{2}\left(U^{(2)}, f^{(2)}\right)\right. & \\ \left.\quad+f_{3}\left(U^{(1)}, f^{(1)}\right)-f_{3}\left(U^{(2)}, f^{(2)}\right)\right) d y_{2} d y_{3}, & \\ \frac{\partial \tilde{p}}{\partial n}=0 & \text { on }(0,1) \times \partial \Omega .\end{cases}
$$


Like 6.1) we have

(6.10)

$$
\begin{aligned}
\| f_{1}\left(U^{(1)}, f^{(1)}\right) & -f_{1}\left(U^{(2)}, f^{(2)}\right) \|_{C^{-1, \alpha}\left(Q^{+}\right)} \\
& +\sum_{k=2}^{4}\left\|f_{k}\left(U^{(1)}, f^{(1)}\right)-f_{k}\left(U^{(2)}, f^{(2)}\right)\right\|_{C^{0, \alpha}\left(Q^{+}\right)} \\
& \leq C(\delta+\eta+\epsilon)\left(\left\|U^{(1)}-U^{(2)}\right\|_{C^{1, \alpha}\left(Q^{+}\right)}+\left\|f^{(1)}-f^{(2)}\right\|_{C^{2, \alpha}(\Omega)}\right) \triangleq C \tilde{e}
\end{aligned}
$$

then (5.10) implies

$$
\|\tilde{p}\|_{C^{1, \alpha}\left(Q^{+}\right)} \leq C \tilde{e} .
$$

Moreover, $\tilde{\vec{u}}_{*}=\left(u_{2}^{(1)}-u_{2}^{(2)}, u_{3}^{(1)}-u_{3}^{(2)}\right)$ satisfies

$$
\left\{\begin{array}{l}
\operatorname{div} \tilde{\vec{u}}_{*}=f_{*}\left(U^{(1)}, f^{(1)}-f_{*}\left(U^{(2)}, f^{(2)}\right) \text { in } \Omega,\right. \\
\operatorname{rot} \tilde{\vec{u}}_{*}=f_{4}\left(U^{(1)}, f^{(1)}-f_{4}\left(U^{(2)}, f^{(2)}\right) \text { in } \Omega,\right. \\
\tilde{u}_{* n}=0 \text { on } \partial \Omega .
\end{array}\right.
$$

By (6.10) we have a similar estimate for $\left\|f_{*}\left(U^{(1)}, f^{(1)}\right)-f_{*}\left(U_{*}^{(2)}, f^{(2)}\right)\right\|_{C^{0, \alpha}\left(Q^{+}\right)}$; then by using (5.20) we have

$$
\left\|\tilde{\vec{u}}_{*}\right\|_{C^{1, \alpha}(\Omega)} \leq C \tilde{e} .
$$

To estimate $\tilde{\vec{\omega}}=\vec{\omega}^{(1)}-\vec{\omega}^{(2)}$, we use (5.16). It is easy to have

$$
\begin{aligned}
\tilde{\vec{\omega}}= & \sum_{j}\left(F_{k j}^{(1)}\left(y_{1}, \zeta_{2}^{(1)}\left(y_{1}, y_{2}, y_{3}\right), \zeta_{3}^{(1)}\left(y_{1}, y_{2}, y_{3}\right)\right) \omega_{j 0}^{(1)}\left(y_{2}, y_{3}\right)\right. \\
& \quad-\left(F_{k j}^{(2)}\left(y_{1}, \zeta_{2}^{(2)}\left(y_{1}, y_{2}, y_{3}\right), \zeta_{3}^{(2)}\left(y_{1}, y_{2}, y_{3}\right)\right)\right) \omega_{j 0}^{(2)}\left(y_{2}, y_{3}\right) \\
= & \sum_{j}\left(F_{k j}^{(1)}-F_{k j}^{(2)}\right) \omega_{j 0}^{(1)}+\sum_{j} F_{k j}^{(2)}\left(\omega_{j 0}^{(1)}-\omega_{j 0}^{(2)}\right) .
\end{aligned}
$$

Then for $U \in O_{\delta}, f \in \Sigma_{\eta}$, (6.15)

$\left\|\tilde{\vec{\omega}}_{k}\right\|_{C^{0, \alpha}\left(Q^{+}\right)} \leq C(\delta+\eta+\epsilon)\left(\left\|U^{(1)}-U^{(2)}\right\|_{C^{1, \alpha}\left(Q^{+}\right)}+\left\|f^{(1)}-f^{(2)}\right\|_{C^{2, \alpha}(\Omega)}\right) \leq C \tilde{e}$.

Finally, for the right hand side of (4.6) we have

$$
\begin{aligned}
& \left\|\nabla\left(-\frac{1}{\rho^{(1)}} \nabla \rho^{(1)} \cdot \vec{u}^{(1)}\right)-\nabla\left(-\frac{1}{\rho^{(2)}} \nabla \rho^{(2)} \cdot \vec{u}^{(2)}\right)\right\|_{C^{-1, \alpha}\left(Q^{+}\right)} \\
& \quad \leq C(\delta+\eta+\epsilon)\left(\left\|U^{(1)}-U^{(2)}\right\|_{C^{1, \alpha}\left(Q^{+}\right)}+\left\|f^{(1)}-f^{(2)}\right\|_{C^{2, \alpha}(\Omega)}\right) \leq C \tilde{e} \\
& \left\|\nabla \times \vec{\omega}^{(1)}-\nabla \times \vec{\omega}^{(2)}\right\|_{C^{-1, \alpha}\left(Q^{+}\right)} \leq\|\tilde{\vec{\omega}}\|_{C^{0, \alpha}\left(Q^{+}\right)} \leq C \tilde{e} \\
& \left\|-\frac{1}{\rho^{(1)}} \nabla p^{(1)}+\frac{1}{\rho^{(2)}} \nabla p^{(2)}\right\|_{C^{0, \alpha}\left(S_{1}\right)} \leq C \tilde{e}
\end{aligned}
$$

Then by using (4.6) we also obtain

$$
\|\tilde{\vec{u}}\|_{C^{1, \alpha}\left(Q^{+}\right)} \leq C \tilde{e} .
$$


Summing up, for small $\delta, \eta, \epsilon$ we establish the contraction of the mapping $T$, so that the solution to problems $\left(\mathbf{P}_{\mathbf{p}}\right),\left(\mathbf{P}_{*}\right),\left(\mathbf{P}_{\omega}\right),\left(\mathbf{P}_{\mathbf{s}}\right),\left(\mathbf{P}_{\mathbf{u}}\right)$ is obtained by using the principle of contractive mapping. Hence problem (NL) is also solved. That is,

Theorem 6.1. For small $\delta_{0}$ we can take $\eta_{0}$ and $\epsilon_{0}$ sufficiently small, such that for any $U^{-} \in K_{\epsilon}, f \in \Sigma_{\eta}$ with $\epsilon<\epsilon_{0}, \eta<\eta_{0}$, problem (NL) admits a unique solution $U \in O_{\delta}$.

We remark here that the above argument is valid for both cases $f^{(1)}=f^{(2)}=f$ and $f^{(1)} \neq f^{(2)}$. The estimate for the case $f^{(1)} \neq f^{(2)}$ will be employed in the next section.

\section{Solution to the free boundary Value Problem}

As we did in Section 2, the free boundary value problem $\left(\mathbf{F B}_{\mathbf{1}}\right)$ is decomposed as the fixed boundary value problem (NL), which has been solved in the last section and is a problem to update the location of the shock front. The latter is problem (3.5), which involves a system with two first order equations. Since the solution $U$ of (NL) satisfies the boundary condition $G_{4}=0$ (see (2.27)), then for any given $U \in O_{\delta}$, the problem

$$
\left\{\begin{array}{l}
\frac{\partial f^{\#}}{\partial x_{i}}=-\frac{\Phi_{i}}{\Phi_{1}}, \quad i=2,3, \\
f^{\#}(0,0)=0,
\end{array}\right.
$$

has a unique solution. Now for any given $f \in \Sigma_{\eta}$ with $\eta \leq \eta_{0}$, we find a solution $U$ of problem (NL) in $O_{\delta}$ by using the result of the last solution. Then from (17.1) we obtain $f^{\#}$.

Notice that for sufficiently small $\delta$ and $\epsilon$,

$$
\begin{aligned}
{[p]=} & p-p_{-}=\left(p-p_{+}^{b}\right)+\left(p_{+}^{b}-p_{-}^{b}\right)+\left(p_{-}^{b}-p_{-}\right) \\
& \geq p_{+}^{b}-p_{-}^{b}-C \epsilon-\delta \geq \frac{1}{2}\left(p_{+}^{b}-p_{-}^{b}\right)
\end{aligned}
$$

then (2.19) implies

$$
\left|\Phi_{1}\right| \geq \frac{1}{8}\left(p_{+}^{b}-p_{-}^{b}\right)^{2}
$$

Meanwhile,

$$
\left\|\left[\rho u_{2}^{2}\right],\left[\rho u_{3}^{2}\right],\left[\rho u_{1} u_{2}\right],\left[\rho u_{1} u_{3}\right],\left[\rho u_{2} u_{3}\right]\right\|_{C^{1, \alpha}\left(S_{0}\right)} \leq C(\delta+\epsilon) .
$$

Hence

$$
\left\|f^{\#}\right\|_{C^{2, \alpha}\left(S_{0}\right)} \leq\left\|\Phi_{2} / \Phi_{1}\right\|_{C^{1, \alpha}\left(S_{0}\right)}+\left\|\Phi_{3} / \Phi_{1}\right\|_{C^{1, \alpha}\left(S_{0}\right)} \leq C(\delta+\epsilon) .
$$

For given $\eta$, we choose a sufficiently small $\epsilon$ and $\delta=C_{0} \epsilon$, such that $\|f\|_{C^{2, \alpha}\left(S_{0}\right)} \leq \eta$. Hence $T_{f}: f \mapsto f^{\#}$ is a mapping for $\Sigma_{\eta}$ to $\Sigma_{\eta}$.

One can also prove the contraction of the mapping $T_{f}$. In fact, by taking $f^{(1)}, f^{(2)}$ in $\Sigma_{\eta}$ and solving (7.1) we obtain $f^{\#(j)}=T_{f} f^{(j)}$ with $j=1,2$. Then $\tilde{f}^{\#}=$ $f^{\#^{(1)}}-f^{\#^{(2)}}$ satisfies

$$
\left\{\begin{array}{l}
\frac{\partial \tilde{f}^{\#}}{\partial x_{i}}=-\frac{\Phi_{i}^{(1)}}{\Phi_{1}^{(1)}}+\frac{\Phi_{i}^{(2)}}{\Phi_{1}^{(2)}}, \quad i=2,3, \\
\tilde{f}^{\#}(0,0)=0 .
\end{array}\right.
$$


Since both $\Phi_{1}^{(1)}$ and $\Phi_{1}^{(2)}$ satisfy (6.3) , we have

$$
\left\|-\Phi_{i}^{(1)} / \Phi_{1}^{(1)}+\Phi_{i}^{(2)} / \Phi_{1}^{(2)}\right\|_{C^{1, \alpha}\left(S_{0}\right)} \leq C\left\|U^{(1)}-U^{(2)}\right\|_{C^{1, \alpha}\left(Q^{+}\right)},
$$

where $U^{(j)}$ is the solution of (NL), which corresponds to $f^{(j)}\left(x_{2}, x_{3}\right)$ describing the approximate shock front. Notice that the argument provided in Section 5 is also available for the case $f^{(1)} \neq f^{(2)}$. Therefore, by using the result there we have

$$
\left\|U^{(1)}-U^{(2)}\right\|_{C^{1, \alpha}\left(Q^{+}\right)} \leq C(\delta+\eta+\epsilon)\left(\left\|U^{(1)}-U^{(2)}\right\|_{C^{1, \alpha}\left(Q^{+}\right)}+\left\|f^{(1)}-f^{(2)}\right\|_{C^{2, \alpha}\left(S_{0}\right)}\right) \text {, }
$$

which leads to

$$
\left\|U^{(1)}-U^{(2)}\right\|_{C^{1, \alpha}\left(Q^{+}\right)} \leq C^{\prime}(\delta+\eta+\epsilon)\left\|f^{(1)}-f^{(2)}\right\|_{C^{2, \alpha}\left(S_{0}\right)} .
$$

Combining (7.9), (7.6) and (7.7), we obtain

$$
\left\|f^{\#(1)}-f^{\#(2)}\right\|_{C^{2, \alpha}\left(S_{0}\right)} \leq \frac{1}{2}\left\|f^{(1)}-f^{(2)}\right\|_{C^{2, \alpha}\left(S_{0}\right)},
$$

provided $\delta_{0}, \eta_{0}, \epsilon_{0}$ are chosen sufficiently small. (7.10) obviously implies the existence of the fixed point of the mapping $T_{f}$. Hence the fixed point $f$ together with the corresponding solution $U$ of (3.4) in $Q_{f}$ solve the boundary value problem $\left(\mathbf{F B}_{\mathbf{1}}\right)$. Then according to the analysis in Section 2, problem $(\mathbf{F B})$, as well as the original problem $(\mathbf{P})$, is also solved correspondingly.

\section{REFERENCES}

1. J.D. Cole and L.P. Cook, Transonic aerodynamics, North-Holland, 30 (1986).

2. G.Q. Chen and M. Feldman, Multidimensional Transonic Shocks and Free Boundary Problems for Nonlinear Equations of Mixed Type, Jour. Amer. Math. Soc. 16(2003),461-494. MR 1969202 (2004d:35182)

3. G.Q. Chen and M. Feldman, Steady Transonic Shocks and Free Boundary Problems in Infinite Cylinders for the Euler Equations, Comm. Pure. Appl. Math. 57(2004),310-356. MR2020107 (2004m:35282)

4. S. Canić, B.L. Keyfitz and G.M. Lieberman, A Proof of Existence of Perturbed Steady Transonic Shocks via a Free Boundary Problem, Comm. Pure. Appl. Math. 53(2000),484511. MR1733695(2001m:76056)

5. S. Canić, B. Kerfitz and E.H. Kim, A free boundary problem for unsteady transonic small disturbance equation: transonic regular reflection, Methods and Appl. Anal. 7(2000),313-336. MR.1869288 (2002h:76077)

6. S. Canić, B. Kerfitz and E.H. Kim, A free boundary problem for a quasilinear degenerate elliptic equation: transonic regular reflection, Comm. Pure Appl. Math. 55(2002),71-92. MR.1857880 (2003a:35206)

7. S. Chen, On the initial-boundary value problem for quasilinear symmetric hyperbolic system and applications, Chin. Ann. Math. 1(1980),511-522. MR619598(83f:35073)

8. S. Chen, Stability of oblique shock fronts, Science in China 45(2002),1012-1019. MR.1942915 $(2004 \mathrm{e}: 35151)$

9. S. Chen, Stability of Transonic Shock Fronts in Two-Dimensional Euler Systems, Trans. Amer. Math. Soc. 357(2005), 287-308. MR2098096 (2005h:35278)

10. R. Courant and K.O. Friedrichs, Supersonic Flow and Shock Waves. Interscience Publishers Inc., New York, 1948. MR0029615 (10:637c)

11. H.M. Glaz and T.P. Liu, The asymptotic analysis of wave interactions and numerical calculations of transonic flow, Advances in Appl. Math. 5(1984),111-146. MR747330 (85j:76019)

12. D. Gilbarg and N.S. Trudinger, Elliptic Partial Differential Equation of Second Order, Second edition, Grundlehren der Mathematischen Wissenschaften, 224. Springer, Berlin-New York, 1983. MR 737190 (86c:35035)

13. P. Grisvard, Elliptic Problems in nonsmooth domains, Monographs and Studies in Mathematics 24, Pitman, London 1985. MR775683 (86m:35044)

14. A.G. Kuz'min, Boundary Value Problems for Transonic Flow, John Wiley \& Sons Ltd. (2002). 
15. T.P. Liu, Nonlinear stability and instability of transonic flows through a nozzle, Comm. Math. Phys. 83(1982), 243-260. MR649161 (83f:35014)

16. T.P. Liu, Transonic gas flows in a variable area duct, Arch. Rat. Mech. Anal. 80 (1982),1-18. MR656799 (83h:76050)

17. T.T. Li and W.C. Yu, Boundary value problem for quasilinear hyperbolic system, Duke University Mathematical Series, v.5, 1985.

18. C.S. Morawetz, On the non-existence of continuous transonic flows past profiles. I,II,III, Comm. Pure Appl. Math. 9 (1956),45-68, 10(1957),107-131, 11(1958),129-144. MR0078130 $(17: 1149 \mathrm{~d})$

19. C.S. Morawetz, Non-existence of transonic flows past profiles, Comm. Pure Appl. Math. 17(1964),357-367. MR0184522(32:1994)

20. J. Smoller, Shock Waves and Reaction-diffusion Equations. second edition, Springer-Verlag, New York, 1994. MR1301779 (95g:35002)

21. Z.P. Xin and H.C. Yin, Transonic Shock in a Nozzle I: Two-Dimensional Case, Comm. Pure Appl. Math., 58(2005), 999-1050. MR2143525(2006c:76079)

School of Mathematical Sciences, Fudan University, Shanghai, 200433, People's RePUBLIC OF CHINA

E-mail address: sxchen@public8.sta.net.cn 